\title{
Linear prediction sufficiency in the misspecified linear model
}

\author{
Augustyn Markiewicz \\ Department of Mathematical and Statistical Methods \\ Poznań University of Life Sciences \\ Wojska Polskiego 28, PL-60637 Poznań, Poland \\ email: amark@up.poznan.pl \\ Simo Puntanen 11 \\ Faculty of Natural Sciences \\ FI-33014 University of Tampere, Finland \\ email: simo.puntanen@uta.fi
}

\begin{abstract}
We consider the general linear model $\mathbf{y}=\mathbf{X} \boldsymbol{\beta}+\boldsymbol{\varepsilon}$ supplemented with the new (future) unobservable random vector $\mathbf{y}_{*}$, coming from $\mathbf{y}_{*}=\mathbf{X}_{*} \boldsymbol{\beta}+\boldsymbol{\varepsilon}_{*}$, where the expectation of $\mathbf{y}_{*}$ is $\mathbf{X}_{*} \boldsymbol{\beta}$ and the covariance matrix of $\mathbf{y}_{*}$ is known as well as the cross-covariance matrix between $\mathbf{y}_{*}$ and $\mathbf{y}$. We denote the supplemented model as $\mathscr{M}_{*}$. The misspecified supplemented model is denoted as $\mathscr{M}_{*}$, and the misspecification concerns the covariance part of the setup. Suppose that Fy is linearly sufficient for estimable parametric function $\mathbf{X}_{*} \boldsymbol{\beta}$ under $\mathscr{M}_{*}$. We give necessary and sufficient conditions that $\mathbf{F y}$ continues to be linearly sufficient for $\mathbf{X}_{*} \boldsymbol{\beta}$ under the model $\underline{M}_{*}$. The corresponding properties regarding the linear prediction sufficiency with respect to $\varepsilon_{*}$ and $\mathbf{y}_{*}$ are also studied.
\end{abstract}

Keywords: BLUE, best linear unbiased estimator, BLUP, best linear unbiased predictor, linear model, linear model with new observations, linear sufficiency.

\footnotetext{
${ }^{1}$ Author responsible for correspondence
} 


\section{Introduction}

Let us start with the notation. The symbol $\mathbb{R}^{m \times n}$ denotes the set of $m \times n$ real matrices, while $\mathbf{A}^{\prime}, \mathbf{A}^{-}, \mathbf{A}^{+}, \mathscr{C}(\mathbf{A})$, and $\mathscr{C}(\mathbf{A})^{\perp}$, denote, respectively, the transpose, a generalized inverse, the Moore-Penrose inverse, the column space, and the orthogonal complement of the column space of the matrix $\mathbf{A}$. By $(\mathbf{A}: \mathbf{B})$ we denote the partitioned matrix with $\mathbf{A}_{a \times b}$ and $\mathbf{B}_{a \times c}$ as submatrices. By $\mathbf{A}^{\perp}$ we denote any matrix satisfying $\mathscr{C}\left(\mathbf{A}^{\perp}\right)=\mathscr{C}(\mathbf{A})^{\perp}$. Furthermore, we will write $\mathbf{P}_{\mathbf{A}}=\mathbf{A} \mathbf{A}^{+}=\mathbf{A}\left(\mathbf{A}^{\prime} \mathbf{A}\right)^{-} \mathbf{A}^{\prime}$ to denote the orthogonal projector (with respect to the standard inner product) onto $\mathscr{C}(\mathbf{A})$. The orthogonal projector onto $\mathscr{C}(\mathbf{A})^{\perp}$ is denoted as $\mathbf{Q}_{\mathbf{A}}=\mathbf{I}_{a}-\mathbf{P}_{\mathbf{A}}$, where $\mathbf{I}_{a}$ refers to the $a \times a$ identity matrix and $a$ is the number of rows of $\mathbf{A}$. It appears handy to use the short notation

$$
\mathbf{M}=\mathbf{I}_{n}-\mathbf{P}_{\mathbf{X}}
$$

where $\mathbf{X}_{n \times p}$ refers to the model matrix; see $(1.2)$ below. One choice for $\mathbf{X}^{\perp}$ is of course $\mathbf{M}$.

Our focus lies in the general linear model

$$
\mathbf{y}=\mathbf{X} \boldsymbol{\beta}+\boldsymbol{\varepsilon}, \text { or shortly } \mathscr{M}=\{\mathbf{y}, \mathbf{X} \boldsymbol{\beta}, \mathbf{V}\},
$$

where $\mathbf{X}_{n \times p}$ is a known model matrix, the vector $\mathbf{y}$ is an observable $n$-dimensional random vector, $\boldsymbol{\beta}$ is a $p \times 1$ vector of unknown parameters, and $\varepsilon$ is an unobservable vector of random errors with expectation $\mathrm{E}(\varepsilon)=\mathbf{0}$, and covariance matrix $\operatorname{cov}(\varepsilon)=$ $\mathbf{V}$. The nonnegative definite matrix $\mathbf{V}$ is known and can be singular.

Let $\mathbf{y}_{*}$ denote a $q \times 1$ unobservable random vector containing new observations. The new observations are assumed to be generated from

$$
\mathbf{y}_{*}=\mathbf{X}_{*} \boldsymbol{\beta}+\varepsilon_{*},
$$

where $\mathbf{X}_{*}$ is a known $q \times p$ matrix, $\boldsymbol{\beta}$ is the same vector of fixed (unknown) parameters as in $\mathscr{M}$, and $\varepsilon_{*}$ is a $q$-dimensional random error vector. We further have

$$
\mathrm{E}\left(\begin{array}{c}
\mathbf{y} \\
\mathbf{y}_{*}
\end{array}\right)=\left(\begin{array}{c}
\mathbf{X} \\
\mathbf{X}_{*}
\end{array}\right) \boldsymbol{\beta}, \quad \operatorname{cov}\left(\begin{array}{c}
\mathbf{y} \\
\mathbf{y}_{*}
\end{array}\right)=\left(\begin{array}{cc}
\mathbf{V} & \mathbf{V}_{12} \\
\mathbf{V}_{21} & \mathbf{V}_{22}
\end{array}\right)=\boldsymbol{\Gamma}
$$

where the covariance matrix $\boldsymbol{\Gamma}$ is known. We denote this setup shortly as

$$
\mathscr{M}_{*}=\left\{\left(\begin{array}{c}
\mathbf{y} \\
\mathbf{y}_{*}
\end{array}\right),\left(\begin{array}{c}
\mathbf{X} \\
\mathbf{X}_{*}
\end{array}\right) \boldsymbol{\beta},\left(\begin{array}{cc}
\mathbf{V} & \mathbf{V}_{12} \\
\mathbf{V}_{21} & \mathbf{V}_{22}
\end{array}\right)\right\} .
$$

We call $\mathscr{M}_{*}$ "the linear model with new observations".

A parametric function $\boldsymbol{\mu}_{*}=\mathbf{X}_{*} \boldsymbol{\beta}$ is said to be estimable if it has a linear unbiased estimator $\mathbf{C y}$. Such a matrix $\mathbf{C} \in \mathbb{R}^{q \times n}$ exists only when $\mathscr{C}\left(\mathbf{X}_{*}^{\prime}\right) \subseteq \mathscr{C}\left(\mathbf{X}^{\prime}\right)$. The linear unbiased estimator $\mathbf{C y}$ is the best linear unbiased estimator, BLUE, of estimable $\mathbf{X}_{*} \boldsymbol{\beta}$ if $\mathbf{C y}$ has the smallest covariance matrix in the Löwner sense among all linear unbiased estimators of $\mathbf{X}_{*} \boldsymbol{\beta}$ :

$$
\operatorname{cov}(\mathbf{C y}) \leq_{\mathrm{L}} \operatorname{cov}\left(\mathbf{C}_{\#} \mathbf{y}\right) \text { for all } \mathbf{C}_{\#}: \mathbf{C}_{\#} \mathbf{X}=\mathbf{X}_{*} .
$$


Correspondingly, the linear predictor $\mathbf{B y}$ is said to be unbiased for $\mathbf{y}_{*}$ if the expected prediction error is zero, i.e., $\mathrm{E}\left(\mathbf{y}_{*}-\mathbf{B y}\right)=\mathbf{0}$ for all $\boldsymbol{\beta} \in \mathbb{R}^{p}$, which happens if and only if $\mathbf{X}_{*}^{\prime}=\mathbf{X}^{\prime} \mathbf{B}^{\prime}$. When $\mathscr{C}\left(\mathbf{X}_{*}^{\prime}\right) \subseteq \mathscr{C}\left(\mathbf{X}^{\prime}\right)$ holds, we will say that $\mathbf{y}_{*}$ is predictable under $\mathscr{M}_{*}$, that is, $\mathbf{y}_{*}$ is predictable whenever $\mathbf{X}_{*} \boldsymbol{\beta}$ is estimable. Now a linear unbiased predictor By is the best linear unbiased predictor, BLUP, for $\mathbf{y}_{*}$, if we have the Löwner ordering

$$
\operatorname{cov}\left(\mathbf{y}_{*}-\mathbf{B y}\right) \leq_{\mathrm{L}} \operatorname{cov}\left(\mathbf{y}_{*}-\mathbf{B}_{\#} \mathbf{y}\right) \text { for all } \mathbf{B}_{\#}: \mathbf{B}_{\#} \mathbf{X}=\mathbf{X}_{*} .
$$

Consider then the BLUP of $\varepsilon_{*}$. Obviously Dy is an unbiased predictor for $\varepsilon_{*}$ if and only if $\mathbf{D X}=\mathbf{0}$, i.e., $\mathbf{D}=\mathbf{L} \mathbf{M}$ for some $\mathbf{L}$. Thus $\mathbf{D y}$ is the BLUP for $\boldsymbol{\varepsilon}_{*}$ if and only if

$$
\operatorname{cov}\left(\varepsilon_{*}-\mathbf{D} \mathbf{y}\right) \leq_{\mathrm{L}} \operatorname{cov}\left(\boldsymbol{\varepsilon}_{*}-\mathbf{D}_{\#} \mathbf{y}\right) \text { for all } \mathbf{D}_{\#}: \mathbf{D}_{\#} \mathbf{X}=\mathbf{0} .
$$

For Lemma 1.1, characterizing the BLUE, see, e.g., Rao (1973, p. 282), and the BLUP, see, e.g., Christensen (2011, p. 294), Isotalo \& Puntanen (2006b, p. 1015), and Haslett \& Puntanen (2017).

Lemma 1.1. Consider the linear model with new observations defined as $\mathscr{M}_{*}$ in $(1.5)$, where $\mathscr{C}\left(\mathbf{X}_{*}^{\prime}\right) \subseteq \mathscr{C}\left(\mathbf{X}^{\prime}\right)$, i.e., $\mathbf{y}_{*}$ is predictable. Then the following statements hold.

(a) Ay is the BLUP for $\mathbf{y}_{*}$ if and only if

$$
\mathbf{A}\left(\mathbf{X}: \mathbf{V X}^{\perp}\right)=\left(\mathbf{X}_{*}: \mathbf{V}_{21} \mathbf{X}^{\perp}\right) \text {. }
$$

(b) By is the BLUE of $\boldsymbol{\mu}_{*}=\mathbf{X}_{*} \boldsymbol{\beta}$ if and only if

$$
\mathbf{B}\left(\mathbf{X}: \mathbf{V X}^{\perp}\right)=\left(\mathbf{X}_{*}: \mathbf{0}\right) \text {. }
$$

In particular, $\mathbf{C y}$ is the BLUE for $\boldsymbol{\mu}=\mathbf{X} \boldsymbol{\beta}$ if and only if

$$
\mathbf{C}\left(\mathbf{X}: \mathbf{V X}^{\perp}\right)=(\mathbf{X}: \mathbf{0}) \text {. }
$$

(c) Dy is the BLUP for $\varepsilon_{*}$ if and only if

$$
\mathbf{D}\left(\mathbf{X}: \mathbf{V X}^{\perp}\right)=\left(\mathbf{0}: \mathbf{V}_{21} \mathbf{X}^{\perp}\right) .
$$

Lemma 2.2.4 of Rao \& Mitra (1971) gives the condition under which the matrix product $\mathbf{A B}^{-} \mathbf{C}$ is invariant with respect to the choice of $\mathbf{B}^{-}$. It says that for nonnull matrices $\mathbf{A}$ and $\mathbf{C}$ the following holds:

$$
\mathbf{A B}^{-} \mathbf{C}=\mathbf{A} \mathbf{B}^{+} \mathbf{C} \Longleftrightarrow \mathscr{C}(\mathbf{C}) \subseteq \mathscr{C}(\mathbf{B}) \& \mathscr{C}\left(\mathbf{A}^{\prime}\right) \subseteq \mathscr{C}\left(\mathbf{B}^{\prime}\right) .
$$

Some (not necessarily unique) solutions to $\mathbf{A}, \mathbf{B}, \mathbf{C}$ and $\mathbf{D}$ in Lemma 1.1 are the following:

$$
\begin{aligned}
& \mathbf{B}=\mathbf{X}_{*}\left(\mathbf{X}^{\prime} \mathbf{W}^{-} \mathbf{X}\right)^{-} \mathbf{X}^{\prime} \mathbf{W}^{-}:=\mathbf{P}_{\mathbf{X}_{*} ; \mathbf{W}^{-}} \\
& \mathbf{C}=\mathbf{X}\left(\mathbf{X}^{\prime} \mathbf{W}^{-} \mathbf{X}\right)^{-} \mathbf{X}^{\prime} \mathbf{W}^{-}:=\mathbf{P}_{\mathbf{X} ; \mathbf{W}^{-}} \\
& \mathbf{D}=\mathbf{V}_{21} \mathbf{M}(\mathbf{M V M})^{-} \mathbf{M} \\
& \mathbf{A}=\mathbf{B}+\mathbf{D}
\end{aligned}
$$


where $\mathbf{W}$ is a matrix belonging to the set $\mathcal{W}$ of nonnegative definite matrices defined as

$$
\mathcal{W}=\left\{\mathbf{W} \in \mathbb{R}^{n \times n}: \mathbf{W}=\mathbf{V}+\mathbf{X} \mathbf{U}^{\prime} \mathbf{X}^{\prime}, \mathscr{C}(\mathbf{W})=\mathscr{C}(\mathbf{X}: \mathbf{V})\right\} .
$$

In $1.15 \mathbf{U}$ can be any matrix comprising $p$ rows as long as $\mathscr{C}(\mathbf{W})=\mathscr{C}(\mathbf{X}: \mathbf{V})$ is satisfied. One obvious choice is $\mathbf{U}=\mathbf{I}_{p}$. . For a review of the properties of $\mathcal{W}$, see, e.g., Puntanen et al. (2011, Sec. 12.3).

We assume the model $\mathscr{M}$ to be consistent in the sense that $\mathbf{y}$ lies in $\mathscr{C}(\mathbf{X}: \mathbf{V})$ with probability 1 . Hence we assume that the observed $\mathbf{y}$ satisfies

$$
\mathbf{y} \in \mathscr{C}(\mathbf{X}: \mathbf{V})=\mathscr{C}\left(\mathbf{X}: \mathbf{V} \mathbf{X}^{\perp}\right)=\mathscr{C}(\mathbf{X}: \mathbf{V M})=\mathscr{C}(\mathbf{X}) \oplus \mathscr{C}(\mathbf{V M})
$$

For the equality $\mathscr{C}(\mathbf{X}: \mathbf{V})=\mathscr{C}(\mathbf{X}: \mathbf{V M})$, we refer to Rao (1974, Lemma 2.1). There is a related decomposition, see, e.g., Puntanen et al. (2011, Th. 8), that is worth mentioning in this context: for any conformable matrices $\mathbf{A}$ and $\mathbf{B}$ we have

$$
\mathscr{C}(\mathbf{A}: \mathbf{B})=\mathscr{C}\left(\mathbf{A}: \mathbf{Q}_{\mathbf{A}} \mathbf{B}\right) .
$$

Let $\mathbf{A}$ and $\mathbf{B}$ be arbitrary $k \times n$ matrices (for some $k$ ). Then, in the consistent model $\mathscr{M}$, the estimators $\mathbf{A y}$ and $\mathbf{B y}$ are said to be equal with probability 1 if

$$
\mathbf{A y}=\mathbf{B y} \quad \text { for all } \mathbf{y} \in \mathscr{C}(\mathbf{X}: \mathbf{V}) \text {. }
$$

In light of $1.14 \mathrm{c}$ we have

$$
\operatorname{BLUP}\left(\varepsilon_{*}\right)=\mathbf{V}_{21} \mathbf{M}(\mathbf{M V M})^{-} \mathbf{M y}
$$

which under the consistent model is invariant for any choice of $(\mathbf{M V M})^{-}$, because for any $\mathbf{y} \in \mathscr{C}(\mathbf{X}: \mathbf{V}), \mathbf{M y} \in \mathscr{C}(\mathbf{M V})=\mathscr{C}(\mathbf{M V M})$. The corresponding uniqueness with respect to all generalized inverses involved holds also for $\operatorname{BLUE}(\mathbf{X} \boldsymbol{\beta})$ and $\operatorname{BLUE}\left(\mathbf{X}_{*} \boldsymbol{\beta}\right)$.

As regards the structure of this paper, in Section 2 we recall some known conditions for the linear sufficiency. In Section 3 we give necessary and sufficient conditions that $\mathbf{F y}$ continues to be linearly sufficient for $\mathbf{X}_{*} \boldsymbol{\beta}$ under the misspecified model $\mathscr{M}$. The misspecification concerns the covariance part of the setup. Baksalary \& Mathew (1986) considered the invariance of the linear sufficiency with respect to $\mathbf{X} \boldsymbol{\beta}$ and they also allowed misspecification in the $\mathbf{X}$-part. In Section 4 we do some particular considerations on linear sufficiency regarding estimable parametric functions, and in Section 5 we give necessary and sufficient conditions that Fy continues to be linearly prediction sufficient for $\varepsilon_{*}$ under the misspecified model $\underline{M}$.

\section{Conditions for linear sufficiency}

A linear statistic $\mathbf{F y}$, where $\mathbf{F} \in \mathbb{R}^{f \times n}$, is called linearly sufficient for estimable $\boldsymbol{\mu}_{*}=\mathbf{X}_{*} \boldsymbol{\beta}$, where $\mathbf{X}_{*} \in \mathbb{R}^{q \times p}$, if there exists a matrix $\mathbf{A} \in \mathbb{R}^{q \times f}$ such that AFy is 
the BLUE for $\mathbf{X}_{*} \boldsymbol{\beta}$. Sometimes we will use the phrase "BLUE-sufficient" and the notation $\mathbf{F y} \in \mathcal{S}\left(\mathbf{X}_{*} \boldsymbol{\beta}\right)$.

The concept of linear prediction sufficiency with respect to $\mathbf{y}_{*}$ and $\varepsilon_{*}$ is defined analogically. Corresponding to the phrase "BLUE-sufficient", we may use the term "BLUP-sufficient".

The concept of linear minimal sufficiency, introduced by Drygas (1983), is defined as follows. A linear statistic Fy is called linearly minimal sufficient if for any other linearly sufficient statistics $\mathbf{S y}$, there exists a matrix $\mathbf{A}$ such that $\mathbf{F y}=\mathbf{A S y}$ almost surely. Notation $\mathbf{F y} \in \mathcal{S}_{0}\left(\mathbf{X}_{*} \boldsymbol{\beta}\right)$ indicates that $\mathbf{F y}$ is linearly minimal sufficient for $\mathbf{X}_{*} \beta$.

For the following Lemma, see, e.g., Baksalary \& Kala (1981, 1986), Drygas (1983), Tian \& Puntanen (2009, Th. 2.8), Kala, Puntanen \& Tian (2017, Th. 2), Isotalo \& Puntanen (2006b), and Isotalo et al. (2018).

Lemma 2.1. Consider the linear model with new observations defined as $\mathscr{M}_{*}$ in (1.5), where $\mathscr{C}\left(\mathbf{X}_{*}^{\prime}\right) \subseteq \mathscr{C}\left(\mathbf{X}^{\prime}\right)$, i.e., $\mathbf{y}_{*}$ is predictable, and let $\mathbf{W} \in \mathcal{W}$.

(a) $\mathbf{F y} \in \mathcal{S}\left(\mathbf{X}_{*} \boldsymbol{\beta}\right)$ if and only if any of the following equivalent conditions holds:
(i) $\mathscr{C}\left(\begin{array}{c}\mathbf{X}_{*}^{\prime} \\ \mathbf{0}\end{array}\right) \subseteq \mathscr{C}\left(\begin{array}{c}\mathbf{X}^{\prime} \mathbf{F}^{\prime} \\ \mathbf{M V \mathbf { F } ^ { \prime }}\end{array}\right)$,
(ii) $\mathscr{C}\left[\mathbf{X}\left(\mathbf{X}^{\prime} \mathbf{W}^{-} \mathbf{X}\right)^{-} \mathbf{X}_{*}^{\prime}\right] \subseteq \mathscr{C}\left(\mathbf{W F}^{\prime}\right)$.

(b) $\mathbf{F y} \in \mathcal{S}(\mathbf{X} \boldsymbol{\beta})$ if and only if any of the following equivalent statements holds:
(iii) $\mathscr{C}\left(\begin{array}{c}\mathbf{X}^{\prime} \\ \mathbf{0}\end{array}\right) \subseteq \mathscr{C}\left(\begin{array}{c}\mathbf{X}^{\prime} \mathbf{F}^{\prime} \\ \mathbf{M V \mathbf { V } ^ { \prime }}\end{array}\right)$,
(iv) $\mathscr{C}(\mathbf{X}) \subseteq \mathscr{C}\left(\mathbf{W} \mathbf{F}^{\prime}\right)$

(c) $\mathbf{F y} \in \mathcal{S}\left(\varepsilon_{*}\right)$ if and only if any of the following equivalent conditions holds:
(v) $\mathscr{C}\left(\begin{array}{c}\mathbf{0} \\ \mathbf{M} \mathbf{V}_{12}\end{array}\right) \subseteq \mathscr{C}\left(\begin{array}{c}\mathbf{X}^{\prime} \mathbf{F}^{\prime} \\ \mathbf{M V F}^{\prime}\end{array}\right)$,
(vi) $\mathscr{C}\left(\mathbf{M V}_{12}\right) \subseteq \mathscr{C}\left(\mathbf{M V F}^{\prime} \mathbf{Q}_{\mathbf{F X}}\right)$

(d) $\mathbf{F y} \in \mathcal{S}\left(\mathbf{y}_{*}\right)$ if and only if

(vii) $\mathscr{C}\left(\begin{array}{c}\mathbf{X}_{*}^{\prime} \\ \mathbf{M V}_{12}\end{array}\right) \subseteq \mathscr{C}\left(\begin{array}{c}\mathbf{X}^{\prime} \mathbf{F}^{\prime} \\ \mathbf{M V} \mathbf{F}^{\prime}\end{array}\right)$

Moreover, the linear minimal sufficiency above is obtained if and only if the corresponding inclusion is equality.

Condition (vi) of Lemma 2.1, in particular the matrix $\mathbf{F}^{\prime} \mathbf{Q}_{\mathbf{F X}}$, requires some further comments. It appears that

$$
\mathscr{C}\left(\mathbf{F}^{\prime} \mathbf{Q}_{\mathbf{F X}}\right)=\mathscr{C}\left(\mathbf{F}^{\prime}\right) \cap \mathscr{C}(\mathbf{M}),
$$

and denoting

$$
\mathbf{N}=\mathbf{P}_{\mathbf{F}^{\prime} \mathbf{Q}_{\mathbf{F X}}}=\mathbf{P}_{\mathscr{C}\left(\mathbf{F}^{\prime}\right) \cap \mathscr{C}(\mathbf{M})},
$$


we see that

$$
\mathbf{M F}^{\prime} \mathbf{Q}_{\mathbf{F X}}=\mathbf{F}^{\prime} \mathbf{Q}_{\mathbf{F X}} \text {, and } \mathbf{M N}=\mathbf{N} .
$$

For properties like 2.1)-(2.3), see Markiewicz \& Puntanen (2018b, Sec. 2).

The following lemma characterizes the mutual relations of the linear sufficiency of $\mathbf{F y}$ for $\mathbf{X}_{*} \boldsymbol{\beta}, \boldsymbol{\varepsilon}_{*}$, and $\mathbf{y}_{*}$.

Lemma 2.2. Consider the following three statements:

(a) $\mathbf{F y}$ is BLUE-sufficient for $\mathbf{X}_{*} \boldsymbol{\beta}$.

(b) Fy is BLUP-sufficient for $\boldsymbol{\varepsilon}_{*}$.

(c) Fy is BLUP-sufficient for $\mathbf{y}_{*}$.

Then above, any two conditions together imply the third one.

We may mention that a necessary and sufficient condition for the implication (c) $\Longrightarrow$ (a) \& (b) can be introduced; see Markiewicz \& Puntanen 2018a, Th. 5).

\section{Linear sufficiency in the misspecified linear model}

Consider the models $\mathscr{M}_{*}$ and $\mathscr{M}_{*}$, where $\mathscr{C}\left(\mathbf{X}_{*}^{\prime}\right) \subseteq \mathscr{C}\left(\mathbf{X}^{\prime}\right)$ (this assumption is holding in all our considerations):

$$
\begin{aligned}
& \mathscr{M}_{*}=\left\{\left(\begin{array}{c}
\mathbf{y} \\
\mathbf{y}_{*}
\end{array}\right),\left(\begin{array}{c}
\mathbf{X} \\
\mathbf{X}_{*}
\end{array}\right) \boldsymbol{\beta},\left(\begin{array}{cc}
\mathbf{V} & \mathbf{V}_{12} \\
\mathbf{V}_{21} & \mathbf{V}_{22}
\end{array}\right)\right\}, \\
& \mathscr{M}_{*}=\left\{\left(\begin{array}{c}
\mathbf{y} \\
\mathbf{y}_{*}
\end{array}\right),\left(\begin{array}{c}
\mathbf{X} \\
\mathbf{X}_{*}
\end{array}\right) \boldsymbol{\beta},\left(\begin{array}{cc}
\underline{\mathbf{V}} & \underline{\mathbf{V}}_{12} \\
\underline{\mathbf{V}}_{21} & \underline{\mathbf{V}}_{22}
\end{array}\right)\right\} .
\end{aligned}
$$

Thus the difference appears only in the covariance matrices

$$
\boldsymbol{\Gamma}=\left(\begin{array}{cc}
\mathbf{V} & \mathbf{V}_{12} \\
\mathbf{V}_{21} & \mathbf{V}_{22}
\end{array}\right), \quad \underline{\boldsymbol{\Gamma}}=\left(\begin{array}{cc}
\underline{\mathbf{V}} & \underline{\mathbf{V}}_{12} \\
\underline{\mathbf{V}}_{21} & \underline{\mathbf{V}}_{22}
\end{array}\right)
$$

By $\mathscr{M}$ and $\mathscr{M}$ we mean the models $\mathscr{M}=\{\mathbf{y}, \mathbf{X} \boldsymbol{\beta}, \mathbf{V}\}$ and $\mathscr{M}=\{\mathbf{y}, \mathbf{X} \boldsymbol{\beta}, \underline{\mathbf{V}}\}$.

Suppose that $\mathbf{F y}$ is linearly sufficient for $\mathbf{X}_{*} \boldsymbol{\beta}$ under $\mathscr{M}_{*}$, i.e.,

$$
\mathbf{F y} \in \mathcal{S}\left(\mathbf{X}_{*} \boldsymbol{\beta} \mid \mathscr{M}_{*}\right) .
$$

Notice that obviously

$$
\mathcal{S}\left(\mathbf{X}_{*} \boldsymbol{\beta} \mid \mathscr{M}_{*}\right)=\mathcal{S}\left(\mathbf{X}_{*} \boldsymbol{\beta} \mid \mathscr{M}\right) .
$$

We can now pose the following question: what is the condition that the same $\mathbf{F y}$ continues to be linearly sufficient under the misspecified model $\mathscr{M}_{*}$, i.e.,

$$
\mathcal{S}\left(\mathbf{X}_{*} \boldsymbol{\beta} \mid \mathscr{M}\right) \subseteq \mathcal{S}\left(\mathbf{X}_{*} \boldsymbol{\beta} \mid \underline{\mathscr{M}}\right) ?
$$


Observe that in view of $(3.4)$ we have above dropped off the subscript $*$ from $\mathscr{M}$ and $\underline{M}$.

We use the following notations:

$$
\begin{aligned}
& \mathcal{W}=\left\{\mathbf{W} \in \mathbb{R}^{n \times n}: \mathbf{W}=\mathbf{V}+\mathbf{X} \mathbf{U} \mathbf{U}^{\prime} \mathbf{X}^{\prime}, \mathscr{C}(\mathbf{W})=\mathscr{C}(\mathbf{X}: \mathbf{V})\right\}, \\
& \underline{\mathcal{W}}=\left\{\underline{\mathbf{W}} \in \mathbb{R}^{n \times n}: \mathbf{W}=\underline{\mathbf{V}}+\mathbf{X} \underline{\mathbf{U}} \underline{\mathbf{U}}^{\prime} \mathbf{X}^{\prime}, \mathscr{C}(\underline{\mathbf{W}})=\mathscr{C}(\mathbf{X}: \underline{\mathbf{V}})\right\} .
\end{aligned}
$$

Thus, in light of part (ii) of Lemma 2.1, the claim (3.5) above can be expressed as

$$
\mathscr{C}(\mathbf{Z}) \subseteq \mathscr{C}\left(\mathbf{W F}^{\prime}\right) \Longrightarrow \mathscr{C}(\underline{\mathbf{Z}}) \subseteq \mathscr{C}\left(\underline{\mathbf{W}} \mathbf{F}^{\prime}\right),
$$

where $\mathbf{W} \in \mathcal{W}, \underline{\mathbf{W}} \in \underline{\mathcal{W}}$, and

$$
\mathbf{Z}=\mathbf{X}\left(\mathbf{X}^{\prime} \mathbf{W}^{-} \mathbf{X}\right)^{-} \mathbf{X}_{*}^{\prime}, \quad \underline{\mathbf{Z}}=\mathbf{X}\left(\mathbf{X}^{\prime} \underline{\mathbf{W}}^{-} \mathbf{X}\right)^{-} \mathbf{X}_{*}^{\prime} .
$$

Correspondingly, in view of part (vi) of Lemma 2.1, $\mathcal{S}\left(\varepsilon_{*} \mid \mathscr{M}\right) \subseteq \mathcal{S}\left(\varepsilon_{*} \mid \underline{M}\right)$ can be expressed as

$$
\mathscr{C}\left(\mathbf{M V}_{12}\right) \subseteq \mathscr{C}\left(\mathbf{M V F} \mathbf{Q}_{\mathbf{F X}}\right) \Longrightarrow \mathscr{C}\left(\mathbf{M} \underline{\mathbf{V}}_{12}\right) \subseteq \mathscr{C}\left(\mathbf{M} \underline{\mathbf{V F}}^{\prime} \mathbf{Q}_{\mathbf{F X}}\right)
$$

In this section we present a solution to implication (3.7) and in Section 5 we deal with (3.9). Baksalary \& Mathew (1986) considered the inclusion $\mathcal{S}(\mathbf{X} \boldsymbol{\beta} \mid \mathscr{M}) \subset$ $\mathcal{S}(\underline{\mathbf{X}} \boldsymbol{\beta} \mid \underline{\mathscr{M}})$, where $\mathscr{M}=\{\mathbf{y}, \underline{\mathbf{X}} \boldsymbol{\beta}, \underline{\mathbf{V}}\}$ and thus they allowed misspecification also in the $\mathbf{X}$-part.

We begin with three preliminary lemmas. First, using (1.16) and (1.17), it is straightforward to prove the following.

Lemma 3.1. Let $\mathbf{W} \in \mathcal{W}, \underline{\mathbf{W}} \in \underline{\mathcal{W}}$, and let $\mathbf{M}$ be defined as earlier. Then the following results hold:

(a) $\mathscr{C}(\underline{\mathbf{W}}) \subseteq \mathscr{C}(\mathbf{W}) \Longleftrightarrow \mathscr{C}(\underline{\mathbf{V}} \mathbf{M}) \subseteq \mathscr{C}(\mathbf{X}: \mathbf{V M})$,

(b) $\mathscr{C}(\underline{\mathbf{W}}) \subseteq \mathscr{C}(\mathbf{W}) \Longleftrightarrow \mathscr{C}(\mathbf{M} \underline{\mathbf{V}}) \subseteq \mathscr{C}(\mathbf{X}: \mathbf{M V})$,

(c) $\mathscr{C}(\underline{\mathbf{W}}) \subseteq \mathscr{C}(\mathbf{W}) \Longleftrightarrow \mathscr{C}(\mathbf{M} \underline{\mathbf{V}}) \subseteq \mathscr{C}(\mathbf{M V})$,

(d) $\mathscr{C}(\underline{\mathbf{V}} \mathbf{M}) \subseteq \mathscr{C}(\mathbf{V M}) \Longrightarrow \mathscr{C}(\underline{\mathbf{W}}) \subseteq \mathscr{C}(\mathbf{W})$,

(e) for any conformable matrices $\mathbf{A}$ and $\mathbf{B}$,

$$
\mathscr{C}(\mathbf{M A}) \subseteq \mathscr{C}(\mathbf{M B}) \Longleftrightarrow \mathscr{C}(\mathbf{A}) \subseteq \mathscr{C}(\mathbf{X}: \mathbf{B})
$$

The following two lemmas due to Baksalary \& Mathew (1986) are crucial for our main results.

Lemma 3.2. Baksalary \& Mathew (1986, Lemma 3)]

Let $\mathbf{A}, \mathbf{B}, \mathbf{C}$, and $\mathbf{D}$ be conformable matrices such that $\mathscr{C}(\mathbf{C}) \subseteq \mathscr{C}(\mathbf{B})$ and $\mathscr{C}(\mathbf{D}) \subseteq$ $\mathscr{C}(\mathbf{A})$, and $\mathbf{C} \neq \mathbf{0}, \mathbf{D} \neq \mathbf{0}$. Then the inclusion

$$
\mathscr{C}(\mathbf{D}) \subseteq \mathscr{C}\left(\mathbf{A B}^{-} \mathbf{C}\right)
$$

is satisfied irrespective of the choice of a generalized inverse $\mathbf{B}^{-}$if and only if the following two conditions hold: 
(a) $\mathscr{C}(\mathbf{D}) \subseteq \mathscr{C}\left(\mathbf{A B}^{+} \mathbf{C}\right)$,

(b) $\mathscr{C}\left(\mathbf{A}^{\prime}\right) \subseteq \mathscr{C}\left(\mathbf{B}^{\prime}\right)$.

Lemma 3.3. Baksalary \& Mathew (1986, Lemma 5)] Let $\mathbf{W}$ be a matrix belonging to the set $\mathcal{W}$ of nonnegative definite matrices defined as in 1.15 . Then

(a) $\mathscr{C}\left(\mathbf{W}^{+} \mathbf{X}\right)=\mathscr{C}(\mathbf{V M})^{\perp} \cap \mathscr{C}(\mathbf{W})=\mathscr{C}\left(\mathbf{Q}_{\mathbf{V M}} \mathbf{X}\right)$,

(b) $\mathscr{C}\left(\mathbf{W}^{+} \mathbf{X}\right)^{\perp}=\mathscr{C}(\mathbf{V M}: \mathbf{Q} \mathbf{W})$,

(c) $\mathscr{C}(\mathbf{V M})^{\perp}=\mathscr{C}\left(\mathbf{Q}_{\mathbf{V M}} \mathbf{X}\right) \oplus \mathscr{C}(\mathbf{W})^{\perp}$.

For Lemma 3.3, see also Markiewicz \& Puntanen (2018b, Lemma 4).

Now we are ready to present the main result of our paper.

Theorem 3.1. Consider the linear models $\mathscr{M}=\{\mathbf{y}, \mathbf{X} \boldsymbol{\beta}, \mathbf{V}\}$ and $\mathscr{M}=\{\mathbf{y}, \mathbf{X} \boldsymbol{\beta}, \underline{\mathbf{V}}\}$, let $\mathscr{C}\left(\mathbf{X}_{*}^{\prime}\right) \subseteq \mathscr{C}\left(\mathbf{X}^{\prime}\right)$, and denote

$$
\mathbf{Z}=\mathbf{X}\left(\mathbf{X}^{\prime} \mathbf{W}^{-} \mathbf{X}\right)^{-} \mathbf{X}_{*}^{\prime}, \quad \underline{\mathbf{Z}}=\mathbf{X}\left(\mathbf{X}^{\prime} \underline{\mathbf{W}}^{-} \mathbf{X}\right)^{-} \mathbf{X}_{*}^{\prime}
$$

Then the inclusion

$$
\mathcal{S}\left(\mathbf{X}_{*} \boldsymbol{\beta} \mid \mathscr{M}\right) \subseteq \mathcal{S}\left(\mathbf{X}_{*} \boldsymbol{\beta} \mid \underline{M}\right)
$$

holds if and only if the following two conditions hold:

(a) $\mathscr{C}(\underline{\mathbf{Z}}) \subseteq \mathscr{C}\left(\underline{\mathbf{W}} \mathbf{W}^{+} \mathbf{Z}\right)$, i.e., $\left(\mathbf{W}^{+} \mathbf{Z}\right)^{\prime} \mathbf{y} \in \mathcal{S}\left(\mathbf{X}_{*} \boldsymbol{\beta} \mid \underline{M}\right)$,

(b) $\mathscr{C}(\underline{\mathbf{W}}) \subseteq \mathscr{C}(\mathbf{W})$,

where the first inclusion can be equivalently expressed as

(c) $\mathscr{C}\left(\underline{\mathbf{W}}^{+} \underline{\mathbf{Z}}\right) \subseteq \mathscr{C}\left(\mathbf{P}_{\underline{\mathbf{w}}} \mathbf{W}^{+} \mathbf{Z}\right)$,

and the second one as

(d) $\mathscr{C}(\underline{\mathbf{V}}) \subseteq \mathscr{C}(\mathbf{W})$, or equivalently as $\mathscr{C}(\mathbf{M} \underline{\mathbf{V}}) \subseteq \mathscr{C}(\mathbf{M V})$.

Proof. By part (ii) of Lemma 2.1, we know the following:

$$
\begin{aligned}
& \mathbf{F y} \in \mathcal{S}\left(\mathbf{X}_{*} \boldsymbol{\beta} \mid \mathscr{M}\right) \Longleftrightarrow \mathscr{C}(\mathbf{Z}) \subseteq \mathscr{C}\left(\mathbf{W F}^{\prime}\right), \\
& \mathbf{F y} \in \mathcal{S}\left(\mathbf{X}_{*} \boldsymbol{\beta} \mid \underline{\mathscr{M}}\right) \Longleftrightarrow \mathscr{C}(\underline{\mathbf{Z}}) \subseteq \mathscr{C}\left(\underline{\mathbf{W}} \mathbf{F}^{\prime}\right) .
\end{aligned}
$$

Assume now that the inclusion

$$
\mathcal{S}\left(\mathbf{X}_{*} \boldsymbol{\beta} \mid \mathscr{M}\right) \subseteq \mathcal{S}\left(\mathbf{X}_{*} \boldsymbol{\beta} \mid \underline{M}\right)
$$

holds, i.e.,

$$
\mathscr{C}(\mathbf{Z}) \subseteq \mathscr{C}\left(\mathbf{W F}^{\prime}\right)
$$

implies

$$
\mathscr{C}(\underline{\mathbf{Z}}) \subseteq \mathscr{C}\left(\underline{\mathbf{W F}}^{\prime}\right)
$$


We know that

$$
\mathbf{X}_{*}\left(\mathbf{X}^{\prime} \mathbf{W}^{-} \mathbf{X}\right)^{-} \mathbf{X}^{\prime} \mathbf{W}^{-} \mathbf{y}=\mathbf{Z}^{\prime} \mathbf{W}^{-} \mathbf{y}
$$

is one representation for the BLUE of $\mathbf{X}_{*} \boldsymbol{\beta}$. The representation (3.17) is invariant with respect to the choice of all generalized inverses involved. Notice that in (3.17) we have used the properties

$$
\begin{aligned}
\mathbf{Z}^{\prime}=\left[\mathbf{X}\left(\mathbf{X}^{\prime} \mathbf{W}^{-} \mathbf{X}\right)^{-} \mathbf{X}_{*}^{\prime}\right]^{\prime}= & \mathbf{X}_{*}\left[\left(\mathbf{X}^{\prime} \mathbf{W}^{-} \mathbf{X}\right)^{-}\right]^{\prime} \mathbf{X}^{\prime}=\mathbf{X}_{*}\left(\mathbf{X}^{\prime} \mathbf{W}^{-} \mathbf{X}\right)^{-} \mathbf{X}^{\prime} \\
& \left(\mathbf{W}^{-} \mathbf{Z}\right)^{\prime} \mathbf{y}=\mathbf{Z}^{\prime} \mathbf{W}^{-} \mathbf{y}
\end{aligned}
$$

which hold in view of $(1.13)$ and the consistency of the model.

Now trivially $\mathbf{Z}^{\prime} \mathbf{W}^{-} \mathbf{y}$ is linearly sufficient for $\mathbf{X}_{*} \boldsymbol{\beta}$ under $\mathscr{M}$ for any choice of $\mathbf{W}^{-}$; in other words, choosing $\mathbf{F}^{\prime}=\mathbf{W}^{-} \mathbf{Z}$, the condition $(3.15)$ is satisfied. Actually $\mathbf{Z}^{\prime} \mathbf{W}^{-} \mathbf{y}$ is linearly minimal sufficient because with this choice of $\mathbf{F}$ we have the equality

$$
\mathscr{C}(\mathbf{Z})=\mathscr{C}\left(\mathbf{W F}^{\prime}\right)
$$

and thus we can denote $\mathbf{Z}^{\prime} \mathbf{W}^{-} \mathbf{y} \in \mathcal{S}_{0}\left(\mathbf{X}_{*} \boldsymbol{\beta} \mid \mathscr{M}\right)$. By assumption (3.14), this same Fy (for any $\mathbf{W}^{-}$) now belongs to $\mathcal{S}\left(\mathbf{X}_{*} \boldsymbol{\beta} \mid \underline{M}\right)$, which means that we must have

$$
\mathscr{C}(\underline{\mathbf{Z}}) \subseteq \mathscr{C}\left(\underline{\mathbf{W}} \mathbf{W}^{-} \mathbf{Z}\right) .
$$

On account of Lemma 3.2 , the inclusion $(3.20)$ is satisfied for every generalized inverse $\mathbf{W}^{-}$if and only if the following two conditions hold:

$$
\begin{aligned}
\mathscr{C}(\underline{\mathbf{Z}}) & \subseteq \mathscr{C}\left(\underline{\mathbf{W}} \mathbf{W}^{+} \mathbf{Z}\right), \\
\mathscr{C}(\underline{\mathbf{W}}) & \subseteq \mathscr{C}(\mathbf{W}) .
\end{aligned}
$$

Thus we have shown that $(3.14)$ implies $(3.21 \mathrm{a})$ and $(3.21 \mathrm{~b})$.

Conversely, suppose that (3.21a) and $(3.21 \mathrm{~b})$ hold and $\mathbf{F} \mathbf{y} \in \mathcal{S}\left(\mathbf{X}_{*} \boldsymbol{\beta} \mid \mathscr{M}\right)$. Then

$$
\begin{aligned}
\mathscr{C}(\underline{\mathbf{Z}}) \subseteq \mathscr{C}\left(\underline{\mathbf{W}} \mathbf{W}^{+} \mathbf{Z}\right) \subseteq \mathscr{C}\left(\underline{\mathbf{W}} \mathbf{W}^{+} \mathbf{W} \mathbf{F}^{\prime}\right) \\
=\mathscr{C}\left(\underline{\mathbf{W}} \mathbf{P}_{\mathbf{W}} \mathbf{F}^{\prime}\right)=\mathscr{C}\left(\underline{\mathbf{W}} \mathbf{F}^{\prime}\right),
\end{aligned}
$$

where the second inclusion follows from $(3.15)$ and the last equality comes from $(3.21 \mathrm{~b})$. Now 3.22 means that $\mathbf{F y} \in \mathcal{S}\left(\mathbf{X}_{*} \boldsymbol{\beta} \mid \underline{M}\right)$. Thus we have shown that (3.21a) and (3.21b) are also sufficient for

$$
\mathcal{S}\left(\mathbf{X}_{*} \boldsymbol{\beta} \mid \mathscr{M}\right) \subseteq \mathcal{S}\left(\mathbf{X}_{*} \boldsymbol{\beta} \mid \underline{M}\right) .
$$

Notice that premultiplying $3.21 \mathrm{a})$ by $\mathbf{W}^{+}$yields claim (c) of Theorem 3.1, i.e.,

$$
\mathscr{C}\left(\underline{\mathbf{W}}^{+} \underline{\mathbf{Z}}\right) \subseteq \mathscr{C}\left(\mathbf{P}_{\underline{\mathbf{w}}} \mathbf{W}^{+} \mathbf{Z}\right) .
$$

On the other hand, premultiplying (3.24) by $\underline{\mathbf{W}}$ and using $\mathscr{C}(\underline{\mathbf{Z}}) \subseteq \mathscr{C}(\underline{\mathbf{W}})$ and (3.21b gives (3.21a). The equivalence of (c) and (d) follows from Lemma 3.3. Thus the proof is completed. 
The following corollary gives the condition under which the equality in 3.12 holds.

Corollary 3.1. The equality in 3.12 in Theorem 3.1

$$
\mathcal{S}\left(\mathbf{X}_{*} \boldsymbol{\beta} \mid \mathscr{M}\right)=\mathcal{S}\left(\mathbf{X}_{*} \boldsymbol{\beta} \mid \underline{M}\right)
$$

holds if and only if the following two conditions hold:

(a) $\mathscr{C}\left(\mathbf{W}^{+} \mathbf{Z}\right)=\mathscr{C}\left(\underline{\mathbf{W}}^{+} \underline{\mathbf{Z}}\right)$

(b) $\mathscr{C}(\underline{\mathbf{W}})=\mathscr{C}(\mathbf{W})$.

Proof. In view of Theorem 3.1, the equality (3.25) holds if and only if the following three conditions hold:

$$
\begin{aligned}
\mathscr{C}(\underline{\mathbf{Z}}) & \subseteq \mathscr{C}\left(\underline{\mathbf{W}} \mathbf{W}^{+} \mathbf{Z}\right), \\
\mathscr{C}(\mathbf{Z}) & \subseteq \mathscr{C}\left(\mathbf{W} \underline{\mathbf{W}}^{+} \underline{\mathbf{Z}}\right), \\
\mathscr{C}(\underline{\mathbf{W}}) & =\mathscr{C}(\mathbf{W}) .
\end{aligned}
$$

Premultiplying(a) by $\underline{\mathbf{W}}$ and using (b) yields (3.26a) and premultiplying(a) by $\mathbf{W}$ yields (3.26b). Similarly, premultiplying (3.26a) by $\underline{\mathbf{W}}^{+}$gives

$$
\mathscr{C}\left(\underline{\mathbf{W}}^{+} \underline{\mathbf{Z}}\right) \subseteq \mathscr{C}\left(\mathbf{P}_{\underline{\mathbf{W}}} \mathbf{W}^{+} \mathbf{Z}\right)=\mathscr{C}\left(\mathbf{W}^{+} \mathbf{Z}\right) .
$$

Correspondingly, (3.26b) implies $\mathscr{C}\left(\mathbf{W}^{+} \mathbf{Z}\right) \subseteq \mathscr{C}\left(\underline{\mathbf{W}}^{+} \underline{\mathbf{Z}}\right)$ and thus the proof is completed.

Consider then the linear minimal sufficiency. Following the proof of Theorem 3.1 . assume that the inclusion

$$
\mathcal{S}_{0}\left(\mathbf{X}_{*} \boldsymbol{\beta} \mid \mathscr{M}\right) \subseteq \mathcal{S}_{0}\left(\mathbf{X}_{*} \boldsymbol{\beta} \mid \underline{\mathscr{M}}\right)
$$

holds, i.e.,

$$
\mathscr{C}(\mathbf{Z})=\mathscr{C}\left(\mathbf{W F}^{\prime}\right) \Longrightarrow \mathscr{C}(\underline{\mathbf{Z}})=\mathscr{C}\left(\underline{\mathbf{W}} \mathbf{F}^{\prime}\right)
$$

We know that

$$
\mathbf{X}_{*}\left(\mathbf{X}^{\prime} \mathbf{W}^{-} \mathbf{X}\right)^{-} \mathbf{X}^{\prime} \mathbf{W}^{-} \mathbf{y}=\mathbf{Z}^{\prime} \mathbf{W}^{-} \mathbf{y}
$$

is one representation for the BLUE of $\mathbf{X}_{*} \boldsymbol{\beta}$. Now $\mathbf{Z}^{\prime} \mathbf{W}^{-} \mathbf{y}$ is minimally linearly sufficient for $\mathbf{X}_{*} \boldsymbol{\beta}$ under $\mathscr{M}$ for any choice of $\mathbf{W}^{-}$; in other words, choosing $\mathbf{F}^{\prime}=$ $\mathbf{W}^{-} \mathbf{Z}$, gives $\mathscr{C}(\mathbf{Z})=\mathscr{C}\left(\mathbf{W F}^{\prime}\right)$. The same $\mathbf{F y}$ should be minimally linearly sufficient under $\mathscr{M}$, that is, we should have

$$
\mathscr{C}(\underline{\mathbf{Z}})=\mathscr{C}\left(\underline{\mathbf{W}} \mathbf{W}^{-} \mathbf{Z}\right) \quad \text { for every } \mathbf{W}^{-} .
$$

In view of Lemma 3.2 , the equality (3.31) is satisfied for every generalized inverse $\mathbf{W}^{-}$if and only if

$$
\mathscr{C}(\underline{\mathbf{Z}})=\mathscr{C}\left(\underline{\mathbf{W}} \mathbf{W}^{+} \mathbf{Z}\right), \quad \text { and } \quad \mathscr{C}(\underline{\mathbf{W}}) \subseteq \mathscr{C}(\mathbf{W}) .
$$

Thus we have obtained the following corollary. 
Corollary 3.2. The inclusion

$$
\mathcal{S}_{0}\left(\mathbf{X}_{*} \boldsymbol{\beta} \mid \mathscr{M}\right) \subseteq \mathcal{S}_{0}\left(\mathbf{X}_{*} \boldsymbol{\beta} \mid \underline{\mathscr{M}}\right)
$$

holds if and only if the following two conditions hold:

(a) $\mathscr{C}(\underline{\mathbf{Z}})=\mathscr{C}\left(\underline{\mathbf{W}} \mathbf{W}^{+} \mathbf{Z}\right)$,

(b) $\mathscr{C}(\underline{\mathbf{W}}) \subseteq \mathscr{C}(\mathbf{W})$.

Consider the special case when

$$
\mathscr{C}(\underline{\mathbf{W}})=\mathscr{C}(\mathbf{W}) .
$$

Then

$$
\operatorname{rank}\left(\mathbf{Z}^{\prime} \mathbf{W}^{+} \underline{\mathbf{W}}\right)=\operatorname{rank}\left(\mathbf{Z}^{\prime} \mathbf{W}^{+} \mathbf{W}\right)=\operatorname{rank}\left(\mathbf{Z}^{\prime} \mathbf{P}_{\mathbf{W}}\right)=\operatorname{rank}\left(\mathbf{Z}^{\prime}\right),
$$

i.e., $\operatorname{rank}(\underline{\mathbf{Z}})=\operatorname{rank}\left(\underline{\mathbf{W}} \mathbf{W}^{+} \mathbf{Z}\right)$ is always valid when (3.34) holds. This means that under (3.34), $\mathscr{C}(\underline{\mathbf{Z}})$ cannot be a proper subspace of $\mathscr{C}\left(\underline{\mathbf{W}} \mathbf{W}^{+} \mathbf{Z}\right)$, i.e., under (3.34), the inclusion

$$
\mathscr{C}(\underline{\mathbf{Z}}) \subseteq \mathscr{C}\left(\underline{\mathbf{W}} \mathbf{W}^{+} \mathbf{Z}\right)
$$

is equivalent to the equality

$$
\mathscr{C}(\underline{\mathbf{Z}})=\mathscr{C}\left(\underline{\mathbf{W}} \mathbf{W}^{+} \mathbf{Z}\right) .
$$

We can thus conclude that under (3.34), the linear minimal sufficiency continues in the misspecified model precisely when the linear (ordinary) sufficiency continues in the misspecified model.

Corollary 3.3. Suppose that $\mathscr{C}(\underline{\mathbf{W}})=\mathscr{C}(\mathbf{W})$. Then the inclusion

$$
\mathcal{S}_{0}\left(\mathbf{X}_{*} \boldsymbol{\beta} \mid \mathscr{M}\right) \subseteq \mathcal{S}_{0}\left(\mathbf{X}_{*} \boldsymbol{\beta} \mid \underline{\mathscr{M}}\right)
$$

holds if and only if

$$
\mathcal{S}\left(\mathbf{X}_{*} \boldsymbol{\beta} \mid \mathscr{M}\right) \subseteq \mathcal{S}\left(\mathbf{X}_{*} \boldsymbol{\beta} \mid \underline{M}\right) .
$$

Let us see how Theorem 3.1 changes when we put $\mathbf{X}_{*}=\mathbf{X}$. Using

$$
\mathscr{C}\left[\mathbf{X}\left(\mathbf{X}^{\prime} \mathbf{W}^{-} \mathbf{X}\right)^{-} \mathbf{X}^{\prime}\right]=\mathscr{C}\left[\mathbf{X}\left(\mathbf{X}^{\prime} \underline{\mathbf{W}}^{-} \mathbf{X}\right)^{-} \mathbf{X}^{\prime}\right]=\mathscr{C}(\mathbf{X}),
$$

the statement (a) in Theorem 3.1 becomes

$$
\mathscr{C}(\mathbf{X}) \subseteq \mathscr{C}\left(\underline{\mathbf{W}} \mathbf{W}^{+} \mathbf{X}\right) .
$$

Obviously $\operatorname{rank}(\mathbf{X}) \geq \operatorname{rank}\left(\underline{\mathbf{W}} \mathbf{W}^{+} \mathbf{X}\right)$ and hence (3.41) becomes equality:

$$
\mathscr{C}(\mathbf{X})=\mathscr{C}\left(\underline{\mathbf{W}} \mathbf{W}^{+} \mathbf{X}\right) .
$$

Using part (a) of Lemma 3.3 and part (c) of Theorem 3.1, we obtain the following corollary. 
Corollary 3.4. [Baksalary \& Mathew (1986, Th. 1)] The inclusion

$$
\mathcal{S}(\mathbf{X} \boldsymbol{\beta} \mid \mathscr{M}) \subseteq \mathcal{S}(\mathbf{X} \boldsymbol{\beta} \mid \underline{\mathscr{M}})
$$

holds if and only if the following two conditions hold:

(a) $\mathscr{C}(\mathbf{X})=\mathscr{C}\left(\underline{\mathbf{W}} \mathbf{W}^{+} \mathbf{X}\right)$, i.e., $\left(\mathbf{W}^{+} \mathbf{X}\right)^{\prime} \mathbf{y} \in \mathcal{S}(\mathbf{X} \boldsymbol{\beta} \mid \underline{\mathscr{M}})$,

(b) $\mathscr{C}(\underline{\mathbf{W}}) \subseteq \mathscr{C}(\mathbf{W})$,

where the part (a) can be replaced with any of the following equivalent conditions:

(c) $\mathscr{C}\left(\underline{\mathbf{W}}^{+} \mathbf{X}\right)=\mathscr{C}\left(\mathbf{P}_{\underline{\mathbf{W}}} \mathbf{W}^{+} \mathbf{X}\right)$,

(d) $\mathscr{C}\left(\mathbf{Q}_{\underline{\mathbf{V M}}} \mathbf{X}\right)=\mathscr{C}\left(\mathbf{P}_{\underline{\mathbf{W}}} \mathbf{Q}_{\mathbf{V M}} \mathbf{X}\right)$.

Corresponding to Corollary 3.4, Baksalary \& Mathew (1986, Th. 1) considered the inclusion $\mathcal{S}(\mathbf{X} \boldsymbol{\beta} \mid \mathscr{M}) \subseteq \mathcal{S}(\underline{\mathbf{X}} \boldsymbol{\beta} \mid \underline{M})$, where $\mathscr{M}=\{\mathbf{y}, \underline{\mathbf{X}} \boldsymbol{\beta}, \underline{\mathbf{V}}\}$.

The following corollary collects together some related equivalent statements.

Corollary 3.5. Consider the linear models $\mathscr{M}$ and $\mathscr{M}$. Then the inclusion

$$
\mathcal{S}(\mathbf{X} \boldsymbol{\beta} \mid \mathscr{M}) \subseteq \mathcal{S}(\mathbf{X} \boldsymbol{\beta} \mid \underline{M})
$$

holds if and only if any of the following equivalent conditions holds:

(a) $\mathscr{C}(\mathbf{X})=\mathscr{C}\left(\underline{\mathbf{W}} \mathbf{W}^{+} \mathbf{X}\right)$ and $\mathscr{C}(\underline{\mathbf{W}}) \subseteq \mathscr{C}(\mathbf{W})$,

(b) $\mathbf{X}^{\prime} \mathbf{W}^{+} \underline{\mathbf{V}} \mathbf{M}=\mathbf{0}$ and $\mathscr{C}(\underline{\mathbf{W}}) \subseteq \mathscr{C}(\mathbf{W})$,

(c) $\mathscr{C}(\underline{\mathbf{V}} \mathbf{M}) \subseteq \mathscr{C}\left(\mathbf{W}^{+} \mathbf{X}\right)^{\perp}=\mathscr{C}(\mathbf{V M}: \mathbf{Q} \mathbf{W})$ and $\mathscr{C}(\underline{\mathbf{W}}) \subseteq \mathscr{C}(\mathbf{W})$,

(d) $\mathscr{C}\left(\underline{\mathbf{V}} \mathbf{W}^{+} \mathbf{X}\right) \subseteq \mathscr{C}(\mathbf{X})$ and $\mathscr{C}(\underline{\mathbf{W}}) \subseteq \mathscr{C}(\mathbf{W})$,

(e) $\mathbf{X}^{\prime} \mathbf{W}^{-} \underline{\mathbf{V}} \mathbf{M}=\mathbf{0}$ for all $\mathbf{W}^{-}$,

(f) $\mathbf{X}\left(\mathbf{X}^{\prime} \mathbf{W}^{-} \mathbf{X}\right)^{-} \mathbf{X}^{\prime} \mathbf{W}^{+} \mathbf{y}=\operatorname{BLUE}(\mathbf{X} \boldsymbol{\beta} \mid \underline{\mathscr{M}})$ and $\mathscr{C}(\underline{\mathbf{W}}) \subseteq \mathscr{C}(\mathbf{W})$,

(g) $\mathbf{X}\left(\mathbf{X}^{\prime} \mathbf{W}^{-} \mathbf{X}\right)^{-} \mathbf{X}^{\prime} \mathbf{W}^{-} \mathbf{y}=\operatorname{BLUE}(\mathbf{X} \boldsymbol{\beta} \mid \underline{M})$ for all $\mathbf{W}^{-}$.

Proof. It is clear by Corollary 3.5 that (3.44) and claim (a) are equivalent. The equality

$$
\mathscr{C}(\mathbf{X})=\mathscr{C}\left(\underline{\mathbf{W}} \mathbf{W}^{+} \mathbf{X}\right)
$$

holds if and only if

$$
\mathscr{C}\left(\underline{\mathbf{W}} \mathbf{W}^{+} \mathbf{X}\right) \subseteq \mathscr{C}(\mathbf{X}), \quad \text { i.e., } \quad \mathbf{M} \underline{\mathbf{W}} \mathbf{W}^{+} \mathbf{X}=\mathbf{M} \underline{\mathbf{V}} \mathbf{W}^{+} \mathbf{X}=\mathbf{0},
$$

and

$$
\operatorname{rank}(\mathbf{X})=\operatorname{rank}\left(\underline{\mathbf{W}} \mathbf{W}^{+} \mathbf{X}\right)
$$


We observe that (3.46) implies the following:

$$
\begin{aligned}
\operatorname{rank}\left(\mathbf{X}^{\prime} \mathbf{W}^{+} \underline{\mathbf{W}}\right) & =\operatorname{rank}\left[\mathbf{X}^{\prime} \mathbf{W}^{+}(\mathbf{X}: \underline{\mathbf{V}} \mathbf{M})\right] \\
& =\operatorname{rank}\left(\mathbf{X}^{\prime} \mathbf{W}^{+} \mathbf{X}\right) \\
& =\operatorname{rank}(\mathbf{X})
\end{aligned}
$$

This means that statements (3.45) and (3.46) are equivalent, i.e., (a) and (b) are equivalent. The equivalence of (b) (c) and (d) is obvious. For the equality

$$
\mathscr{C}\left(\mathbf{W}^{+} \mathbf{X}\right)^{\perp}=\mathscr{C}\left(\mathbf{V M}: \mathbf{Q}_{\mathbf{W}}\right),
$$

we refer to Lemma 3.3. Consider then claim (e), On account of 1.13, we observe that

$$
\mathbf{X}^{\prime} \mathbf{W}^{-} \underline{\mathbf{V} M}=\mathbf{0} \quad \text { for all } \mathbf{W}^{-}
$$

is satisfied if and only if

$$
\mathbf{X}^{\prime} \mathbf{W}^{+} \underline{\mathbf{V}} \mathbf{M}=\mathbf{0}
$$

holds along with

$$
\mathscr{C}(\underline{\mathbf{V}} \mathbf{M}) \subseteq \mathscr{C}(\mathbf{W}), \quad \text { i.e., } \quad \mathscr{C}(\underline{\mathbf{W}}) \subseteq \mathscr{C}(\mathbf{W}) .
$$

This confirms that (d) and (e) are equivalent.

Denoting

$$
\mathbf{P}_{\mathbf{X} ; \mathbf{W}^{-}}=\mathbf{X}\left(\mathbf{X}^{\prime} \mathbf{W}^{-} \mathbf{X}\right)^{-} \mathbf{X}^{\prime} \mathbf{W}^{-}, \quad \mathbf{P}_{\mathbf{X} ; \mathbf{W}^{+}}=\mathbf{X}\left(\mathbf{X}^{\prime} \mathbf{W}^{-} \mathbf{X}\right)^{-} \mathbf{X}^{\prime} \mathbf{W}^{+},
$$

where $\mathbf{W}^{-}$is an arbitrary but fixed generalized inverse of $\mathbf{W}$, we know that $\mathbf{P}_{\mathbf{X} ; \mathbf{W}}-\mathbf{y}$

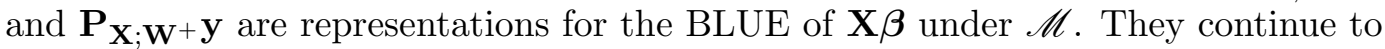
be representations for the BLUE under $\mathscr{M}$ if and only if

$$
\begin{aligned}
& \mathbf{P}_{\mathbf{X} ; \mathbf{W}^{-}} \mathbf{V} \mathbf{M}=\mathbf{X}\left(\mathbf{X}^{\prime} \mathbf{W}^{-} \mathbf{X}\right)^{-} \mathbf{X}^{\prime} \mathbf{W}^{-} \underline{\mathbf{V}} \mathbf{M}=\mathbf{0} \\
& \mathbf{P}_{\mathbf{X} ; \mathbf{W}^{+}} \underline{\mathbf{V}} \mathbf{M}=\mathbf{X}\left(\mathbf{X}^{\prime} \mathbf{W}^{-} \mathbf{X}\right)^{-} \mathbf{X}^{\prime} \mathbf{W}^{+} \underline{\mathbf{V}} \mathbf{M}=\mathbf{0}
\end{aligned}
$$

respectively. Premultiplying (3.54a) and $3.54 \mathrm{~b})$ by $\mathbf{X}^{\prime} \mathbf{W}^{-}$yields the following conclusions:

$$
\begin{aligned}
& \mathbf{P}_{\mathbf{X} ; \mathbf{W}^{-} \mathbf{y}}=\operatorname{BLUE}(\mathbf{X} \boldsymbol{\beta} \mid \underline{M}) \Longleftrightarrow \mathbf{X}^{\prime} \mathbf{W}^{-} \underline{\mathbf{V}} \mathbf{M}=\mathbf{0}, \\
& \mathbf{P}_{\mathbf{X} ; \mathbf{W}^{+}} \mathbf{y}=\operatorname{BLUE}(\mathbf{X} \boldsymbol{\beta} \mid \underline{\mathscr{M}}) \Longleftrightarrow \mathbf{X}^{\prime} \mathbf{W}^{+} \underline{\mathbf{V}} \mathbf{M}=\mathbf{0} .
\end{aligned}
$$

Thus we can conclude that (f) and (g) are equivalent to other claims (a) - (e), For the properties related to (3.55b), see, e.g., Mitra \& Moore (1973, Th. 2.1, Th. 2.2, Note 1), and Hauke et al. (2012, 2013).

Because the property $\mathcal{S}(\mathbf{X} \boldsymbol{\beta} \mid \mathscr{M}) \subseteq \mathcal{S}(\mathbf{X} \boldsymbol{\beta} \mid \mathscr{M})$ is so strongly related to certain BLUE-properties, one may wonder whether the corresponding property for estimable parametric function $\mathbf{X}_{*} \boldsymbol{\beta}$ would hold, i.e., whether the inclusion $\mathcal{S}\left(\mathbf{X}_{*} \boldsymbol{\beta} \mid \mathscr{M}\right) \subseteq$ $\mathcal{S}\left(\mathbf{X}_{*} \boldsymbol{\beta} \mid \underline{M}\right)$ could be described as in (f) and (g) Corollary 3.5 . 
We know that

$$
\mathbf{X}_{*}\left(\mathbf{X}^{\prime} \mathbf{W}^{-} \mathbf{X}\right)^{-} \mathbf{X}^{\prime} \mathbf{W}^{-} \mathbf{y}=\mathbf{Z}^{\prime} \mathbf{W}^{-} \mathbf{y}
$$

is one representation for the $\operatorname{BLUE}\left(\mathbf{X}_{*} \boldsymbol{\beta} \mid \mathscr{M}\right)$ if and only if

$$
\mathbf{Z}^{\prime} \mathbf{W}^{-}(\mathbf{X}: \underline{\mathbf{V}} \mathbf{M})=\left(\mathbf{X}_{*}: \mathbf{0}\right) .
$$

The $\mathbf{X}$-part in (3.57) is obviously holding and hence it only remains to check

$$
\mathbf{Z}^{\prime} \mathbf{W}^{-} \underline{\mathbf{V M}}=\mathbf{0} .
$$

Thus it can be seen at once that the following result holds:

Corollary 3.6. The following statements are equivalent:

(a) $\mathbf{X}_{*}\left(\mathbf{X}^{\prime} \mathbf{W}^{-} \mathbf{X}\right)^{-} \mathbf{X}^{\prime} \mathbf{W}^{-} \mathbf{y}=\operatorname{BLUE}\left(\mathbf{X}_{*} \boldsymbol{\beta} \mid \underline{M}\right)$ for all $\mathbf{W}^{-}$,

(b) $\mathscr{C}\left(\underline{\mathbf{W}} \mathbf{W}^{+} \mathbf{Z}\right) \subseteq \mathscr{C}(\mathbf{X})$ and $\mathscr{C}(\underline{\mathbf{W}}) \subseteq \mathscr{C}(\mathbf{W})$.

Corresponding to (3.53) we may denote

$$
\mathbf{P}_{\mathbf{X}_{*} ; \mathbf{W}^{+}}=\mathbf{X}_{*}\left(\mathbf{X}^{\prime} \mathbf{W}^{-} \mathbf{X}\right)^{-} \mathbf{X}^{\prime} \mathbf{W}^{+}=\mathbf{Z}^{\prime} \mathbf{W}^{+} .
$$

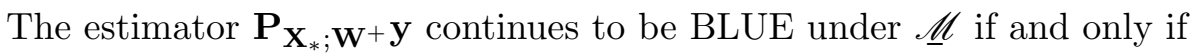

$$
\mathbf{P}_{\mathbf{X}_{*} ; \mathbf{W}^{+}}(\mathbf{X}: \underline{\mathbf{V} M})=\mathbf{Z}^{\prime} \mathbf{W}^{+}(\mathbf{X}: \underline{\mathbf{V}} \mathbf{M})=\left(\mathbf{X}_{*}: \mathbf{0}\right) .
$$

If $\mathbf{F y} \in \mathcal{S}\left(\mathbf{X}_{*} \boldsymbol{\beta} \mid \mathscr{M}\right)$, then by part (ii) of Lemma 2.1, there exists a matrix $\mathbf{C}$ such that $\mathbf{Z}=\mathbf{W} \mathbf{F}^{\prime} \mathbf{C}^{\prime}$. Hence in this case (3.60) becomes

$$
\operatorname{CFP}_{\mathbf{W}}(\mathbf{X}: \underline{\mathbf{V M}})=\left(\mathbf{X}_{*}: \mathbf{0}\right) .
$$

Now if $\mathscr{C}(\mathbf{X}: \underline{\mathbf{V}} \mathbf{M})=\mathscr{C}(\underline{\mathbf{W}}) \subseteq \mathscr{C}(\mathbf{W}),(3.61)$ can be written as

$$
\mathbf{C F}(\mathbf{X}: \underline{\mathbf{V M}})=\left(\mathbf{X}_{*}: \mathbf{0}\right),
$$

which guarantees that $\mathbf{F y} \in \mathcal{S}\left(\mathbf{X}_{*} \boldsymbol{\beta} \mid \underline{M}\right)$. Thus we can conclude the following.

Corollary 3.7. Statement (a) implies (b), where

(a) $\mathbf{X}_{*}\left(\mathbf{X}^{\prime} \mathbf{W}^{-} \mathbf{X}\right)^{-} \mathbf{X}^{\prime} \mathbf{W}^{+} \mathbf{y}=\operatorname{BLUE}\left(\mathbf{X}_{*} \boldsymbol{\beta} \mid \underline{M}\right)$ and $\mathscr{C}(\underline{\mathbf{W}}) \subseteq \mathscr{C}(\mathbf{W})$,

(b) $\left\{\mathcal{S}\left(\mathbf{X}_{*} \boldsymbol{\beta} \mid \mathscr{M}\right)\right\} \subseteq\left\{\mathcal{S}\left(\mathbf{X}_{*} \boldsymbol{\beta} \mid \underline{M}\right)\right\}$.

In Corollary 3.5 we have considered two particular representations of BLUE

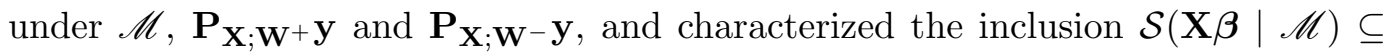
$\mathcal{S}(\mathbf{X} \boldsymbol{\beta} \mid \underline{M})$ via claims (f) and (g). What happens if we request that every representations of BLUE of $\overline{\mathbf{X} \boldsymbol{\beta}}$ under $\mathscr{M}$ continues to be BLUE under $\mathscr{M}$ ? Let a short notation for this be

$$
\{\operatorname{BLUE}(\mathbf{X} \boldsymbol{\beta} \mid \mathscr{M})\} \subseteq\{\operatorname{BLUE}(\mathbf{X} \boldsymbol{\beta} \mid \mathscr{M})\} .
$$


It is well known that $(3.63)$ holds if and only if

$$
\mathscr{C}(\underline{\mathbf{V}} \mathbf{M}) \subseteq \mathscr{C}(\mathbf{V M}) .
$$

For the relation between (3.63) and (3.64), see, e.g., Mitra \& Moore (1973, Th. 4.14.2), Rao (1971, Th. 5.2, Th. 5.5), and Tian (2009). If (3.64) holds, then $\mathscr{C}(\underline{\mathbf{W}}) \subseteq$ $\mathscr{C}(\mathbf{W})$, and we observe immediately that the conditions (a) $(\mathrm{g})$ of Corollary 3.5 hold. Thus (3.64) is a sufficient condition for (3.44). This conclusion could also be drawn by noting that if there exists a matrix $\mathbf{A}$ such that

$$
\mathbf{A F}(\mathbf{X}: \mathbf{V M})=(\mathbf{X}: \mathbf{0}),
$$

then by 3.63 ,

$$
\mathbf{A F}(\mathbf{X}: \underline{\mathbf{V}} \mathbf{M})=(\mathbf{X}: \mathbf{0}),
$$

indicating that (3.44) holds. For clarity, we may write the following:

Corollary 3.8. If every representations of BLUE of $\mathbf{X} \boldsymbol{\beta}$ under $\mathscr{M}$ continues to be BLUE under $\mathscr{M}$, then $\mathbf{F y} \in \mathcal{S}(\mathbf{X} \boldsymbol{\beta} \mid \mathscr{M}) \Longrightarrow \mathbf{F y} \in \mathcal{S}(\mathbf{X} \boldsymbol{\beta} \mid \mathscr{M})$.

\section{Some particular considerations on linear sufficiency regarding estimable parametric functions}

Case 4.1. [Full $\operatorname{rank} \mathbf{X}$ and $\mathscr{C}(\mathbf{X}) \subseteq \mathscr{C}(\mathbf{V})=\mathscr{C}(\underline{\mathbf{V}})$.] Let us consider the special case when $\boldsymbol{\beta}$ itself is estimable and the covariance matrices $\mathbf{V}$ and $\underline{\mathbf{V}}$ have property $\mathscr{C}(\mathbf{V})=\mathscr{C}(\underline{\mathbf{V}})$, and moreover,

$$
\mathscr{C}(\mathbf{X}) \subseteq \mathscr{C}(\mathbf{V})=\mathscr{C}(\underline{\mathbf{V}}) .
$$

Assumption 4.1 means that both $\mathscr{M}$ and $\mathscr{M}$ are so-called weakly singular linear models; see Zyskind \& Martin (1969). In this situation we can choose $\mathbf{W}=\mathbf{V}$ and $\underline{\mathbf{W}}=\underline{\mathbf{V}}$. Now $\mathbf{X}$ has full column rank, $\mathbf{X}_{*}=\mathbf{I}_{p}$, and $\mathscr{C}(\mathbf{Z})=\mathscr{C}(\underline{\mathbf{Z}})=\mathscr{C}(\mathbf{X})$, and thus Theorem 3.1 gives the following corollary.

Corollary 4.1. Let $\mathbf{V}$ and $\underline{\mathbf{V}}$ satisfy $\mathscr{C}(\mathbf{X}) \subseteq \mathscr{C}(\mathbf{V})=\mathscr{C}(\underline{\mathbf{V}})$ and let $\boldsymbol{\beta}$ be estimable. Then

$$
\mathbf{F y} \in \mathcal{S}(\boldsymbol{\beta} \mid \mathscr{M}) \Longleftrightarrow \mathbf{F y} \in \mathcal{S}(\boldsymbol{\beta} \mid \underline{\mathscr{M}})
$$

holds if and only if

$$
\mathscr{C}(\mathbf{X})=\mathscr{C}\left(\underline{\mathbf{V}} \mathbf{V}^{+} \mathbf{X}\right), \text { i.e., }\left(\mathbf{V}^{+} \mathbf{X}\right)^{\prime} \mathbf{y} \in \mathcal{S}(\boldsymbol{\beta} \mid \underline{\mathscr{M}}),
$$

which can be equivalently expressed as

$$
\mathscr{C}\left(\underline{\mathbf{V}}^{+} \mathbf{X}\right)=\mathscr{C}\left(\mathbf{V}^{+} \mathbf{X}\right) .
$$


Markiewicz (1998, Prop. 2) proved Corollary 4.1 assuming that $\mathbf{V}$ and $\underline{\mathbf{V}}$ are positive definite. As one referee pointed out, as a closely related example, we might consider models $\mathscr{M}$ and $\mathscr{M}$, where $\mathbf{1} \in \mathscr{C}(\mathbf{X})$ and the positive definite covariance matrices $\mathbf{V}$ and $\underline{\mathbf{V}}$ have intraclass correlation structures. Then it is well known, see Puntanen \& Styan (1989), that the $\operatorname{BLUE}(\mathbf{X} \boldsymbol{\beta})$ equals $\operatorname{OLSE}(\mathbf{X} \boldsymbol{\beta})$, the ordinary least squares estimator. This further means that the linear sufficiency considerations can be based simply on models where $\mathbf{V}$ and $\underline{\mathbf{V}}$ would be replaced with $\mathbf{I}_{n}$. As a result we would have $\{\mathcal{S}(\mathbf{X} \boldsymbol{\beta} \mid \mathscr{M})\}=\{\mathcal{S}(\mathbf{X} \boldsymbol{\beta} \mid \underline{M})\}$.

Case 4.2. [Partitioned linear model.] Consider then the estimation of $\boldsymbol{\mu}_{1}=$ $\mathbf{X}_{1} \boldsymbol{\beta}_{1}$ under the partitioned model

$$
\mathscr{M}_{12}=\left\{\mathbf{y}, \mathbf{X}_{1} \boldsymbol{\beta}_{1}+\mathbf{X}_{2} \boldsymbol{\beta}_{2}, \mathbf{V}\right\},
$$

where $\mathscr{C}\left(\mathbf{X}_{1}\right) \cap \mathscr{C}\left(\mathbf{X}_{2}\right)=\{\mathbf{0}\}$, so that $\boldsymbol{\mu}_{1}$ is estimable. Now $\mathbf{X}_{*}=\left(\mathbf{X}_{1}: \mathbf{0}\right)$ and in this particular case, we have the following representations for the matrix $\mathbf{Z}$ :

$$
\mathbf{Z}=\mathbf{X}\left(\mathbf{X}^{\prime} \mathbf{W}^{-} \mathbf{X}\right)^{-} \mathbf{X}_{*}^{\prime}=\mathbf{W} \dot{\mathbf{M}}_{2} \mathbf{X}_{1}\left(\mathbf{X}_{1}^{\prime} \dot{\mathbf{M}}_{2} \mathbf{X}_{1}\right)^{-} \mathbf{X}_{1}^{\prime}
$$

where

$$
\dot{\mathbf{M}}_{2}=\mathbf{M}_{2}\left(\mathbf{M}_{2} \mathbf{W} \mathbf{M}_{2}\right)^{-} \mathbf{M}_{2}, \quad \mathbf{M}_{2}=\mathbf{I}_{n}-\mathbf{P}_{\mathbf{X}_{2}},
$$

and column space of $\mathbf{Z}$ is

$$
\mathscr{C}(\mathbf{Z})=\mathscr{C}\left(\mathbf{W} \dot{\mathbf{M}}_{2} \mathbf{X}_{1}\right)
$$

This gives Corollary 4.2 .

Corollary 4.2. Let $\boldsymbol{\mu}_{1}=\mathbf{X}_{1} \boldsymbol{\beta}_{1}$ be estimable under $\mathscr{M}_{12}$ and let $\mathbf{W} \in \mathcal{W}$. Then the statistic $\mathbf{F y}$ is linearly sufficient for $\boldsymbol{\mu}_{1}$ under $\mathscr{M}_{12}$ if and only if

$$
\mathscr{C}\left(\mathbf{W} \dot{\mathbf{M}}_{2} \mathbf{X}_{1}\right) \subseteq \mathscr{C}\left(\mathbf{W} \mathbf{F}^{\prime}\right)
$$

where $\dot{\mathbf{M}}_{2}=\mathbf{M}_{2}\left(\mathbf{M}_{2} \mathbf{W} \mathbf{M}_{2}\right)^{-} \mathbf{M}_{2}$.

The linear sufficiency condition 4.9 was proved by Kala, Markiewicz \& Puntanen (2017, Sec. 3), and, using a different approach, by Isotalo \& Puntanen (2006a, Th. 2).

The inclusion $\mathcal{S}\left(\mathbf{X}_{*} \boldsymbol{\beta} \mid \mathscr{M}_{12}\right) \subseteq \mathcal{S}\left(\mathbf{X}_{*} \boldsymbol{\beta} \mid \mathscr{M}_{12}\right)$ holds if and only if the following two conditions hold:

$$
\begin{aligned}
\mathscr{C}(\underline{\mathbf{Z}}) & \subseteq \mathscr{C}\left(\underline{\mathbf{W}} \mathbf{W}^{+} \mathbf{Z}\right), \\
\mathscr{C}(\underline{\mathbf{W}}) & \subseteq \mathscr{C}(\mathbf{W}) .
\end{aligned}
$$

Putting $\mathbf{X}_{*}=\left(\mathbf{X}_{1}: \mathbf{0}\right)$ gives

$$
\begin{aligned}
& \mathbf{Z}=\dot{\mathbf{W}}_{2} \mathbf{X}_{1}\left(\mathbf{X}_{1}^{\prime} \dot{\mathbf{M}}_{2} \mathbf{X}_{1}\right)^{-} \mathbf{X}_{1}^{\prime}=\mathbf{X}\left(\mathbf{X}^{\prime} \mathbf{W}^{-} \mathbf{X}\right)^{-} \mathbf{X}_{*}^{\prime}, \\
& \underline{\mathbf{Z}}=\underline{\mathbf{W}} \dot{\mathbf{M}}_{2} \mathbf{X}_{1}\left(\mathbf{X}_{1}^{\prime} \dot{\mathbf{M}}_{2} \mathbf{X}_{1}\right)^{-} \mathbf{X}_{1}^{\prime}=\mathbf{X}\left(\mathbf{X}^{\prime} \underline{\mathbf{W}}^{-} \mathbf{X}\right)^{-} \mathbf{X}_{*}^{\prime},
\end{aligned}
$$


with column spaces

$$
\mathscr{C}(\mathbf{Z})=\mathscr{C}\left(\mathbf{W} \dot{\mathbf{M}}_{2} \mathbf{X}_{1}\right), \quad \mathscr{C}(\underline{\mathbf{Z}})=\mathscr{C}\left(\underline{\mathbf{W}} \underline{\mathbf{M}}_{2} \mathbf{X}_{1}\right) .
$$

In this situation (4.10a) becomes

$$
\mathscr{C}\left(\underline{\mathbf{W}} \dot{\mathbf{M}}_{2} \mathbf{X}_{1}\right) \subseteq \mathscr{C}\left(\underline{\mathbf{W}} \mathbf{W}^{+} \mathbf{W} \dot{\mathbf{M}}_{2} \mathbf{X}_{1}\right)=\mathscr{C}\left(\underline{\mathbf{W}} \mathbf{P}_{\mathbf{W}} \dot{\mathbf{M}}_{2} \mathbf{X}_{1}\right)=\mathscr{C}\left(\underline{\mathbf{W}} \dot{\mathbf{M}}_{2} \mathbf{X}_{1}\right)
$$

where we have used 4.10b). Premultiplying 4.14), i.e.,

$$
\mathscr{C}\left[\underline{\mathbf{W}} \mathbf{M}_{2}\left(\mathbf{M}_{2} \underline{\mathbf{W M}}_{2}\right)^{-} \mathbf{M}_{2} \mathbf{X}_{1}\right] \subseteq \mathscr{C}\left[\underline{\mathbf{W}} \mathbf{M}_{2}\left(\mathbf{M}_{2} \mathbf{W M}_{2}\right)^{-} \mathbf{M}_{2} \mathbf{X}_{1}\right]
$$

by $\mathbf{M}_{2}$ yields the equivalent form

$$
\mathscr{C}\left(\mathbf{M}_{2} \mathbf{X}_{1}\right) \subseteq \mathscr{C}\left[\mathbf{M}_{2} \underline{\mathbf{W}} \mathbf{M}_{2}\left(\mathbf{M}_{2} \mathbf{W M}_{2}\right)^{-} \mathbf{M}_{2} \mathbf{X}_{1}\right] .
$$

Thus we have the following result.

Corollary 4.3. Let $\boldsymbol{\mu}_{1}=\mathbf{X}_{1} \boldsymbol{\beta}_{1}$ be estimable under $\mathscr{M}_{12}$ and let $\mathbf{W} \in \mathcal{W}$ and $\underline{\mathbf{W}} \in \underline{\mathcal{W}}$. Then the inclusion

$$
\mathcal{S}\left(\mathbf{X}_{1} \boldsymbol{\beta}_{1} \mid \mathscr{M}_{12}\right) \subseteq \mathcal{S}\left(\mathbf{X}_{1} \boldsymbol{\beta}_{1} \mid \underline{M}_{12}\right)
$$

holds if and only if the following two conditions hold:

(a) $\mathscr{C}\left(\mathbf{M}_{2} \mathbf{X}_{1}\right) \subseteq \mathscr{C}\left[\mathbf{M}_{2} \underline{\mathbf{W}} \mathbf{M}_{2}\left(\mathbf{M}_{2} \mathbf{W} \mathbf{M}_{2}\right)^{-} \mathbf{M}_{2} \mathbf{X}_{1}\right]$,

(b) $\mathscr{C}(\underline{\mathbf{W}}) \subseteq \mathscr{C}(\mathbf{W})$.

\section{Misspecification and the linear sufficiency with re- spect to the error term}

In this section we study when the following holds:

$$
\mathcal{S}\left(\varepsilon_{*} \mid \mathscr{M}_{*}\right) \subseteq \mathcal{S}\left(\varepsilon_{*} \mid \mathscr{M}_{*}\right) \quad ?
$$

In other words,

$$
\mathscr{C}\left(\mathbf{M V}_{12}\right) \subseteq \mathscr{C}\left(\mathbf{M V F}^{\prime} \mathbf{Q}_{\mathbf{F X}}\right) \Longrightarrow \mathscr{C}\left(\mathbf{M} \underline{\mathbf{V}}_{12}\right) \subseteq \mathscr{C}\left(\mathbf{M} \underline{\mathbf{V F}}^{\prime} \mathbf{Q}_{\mathbf{F X}}\right)
$$

The following theorem gives the result.

Theorem 5.1. Consider the linear models (with new observations) $\mathscr{M}_{*}$ and $\underline{M}_{*}$. Then the inclusion

$$
\mathcal{S}\left(\varepsilon_{*} \mid \mathscr{M}_{*}\right) \subseteq \mathcal{S}\left(\varepsilon_{*} \mid \underline{M}_{*}\right)
$$

holds if and only if the following two conditions hold: 
(a) $\mathscr{C}\left(\mathbf{M} \underline{\mathbf{V}}_{12}\right) \subseteq \mathscr{C}\left[\mathbf{M} \underline{\mathbf{V M}}(\mathbf{M V M})^{+} \mathbf{M V} \mathbf{V}_{12}\right]$,

(b) $\mathscr{C}(\underline{\mathbf{W}}) \subseteq \mathscr{C}(\mathbf{W})$

where (a) can be equivalently expressed in the following two forms:

(c) $\mathbf{V}_{21} \mathbf{M}(\mathbf{M V M})^{+} \mathbf{M y} \in \mathcal{S}\left(\varepsilon_{*} \mid \underline{M}_{*}\right)$, i.e., $\operatorname{BLUP}\left(\varepsilon_{*} \mid \mathscr{M}_{*}\right) \in \mathcal{S}\left(\varepsilon_{*} \mid \mathscr{M}_{*}\right)$,

(d) $\mathscr{C}\left(\underline{\mathbf{V}}_{12}\right) \subseteq \mathscr{C}\left[\mathbf{X}: \underline{\mathbf{V M}}(\mathbf{M V M})^{+} \mathbf{M} \mathbf{V}_{12}\right]$

Proof. Assume now that the inclusion

$$
\mathcal{S}\left(\varepsilon_{*} \mid \mathscr{M}_{*}\right) \subseteq \mathcal{S}\left(\varepsilon_{*} \mid \mathscr{M}_{*}\right)
$$

holds, which, according to part (vi) of Lemma 2.1, means that

$$
\mathscr{C}\left(\mathbf{M V}_{12}\right) \subseteq \mathscr{C}\left(\mathbf{M V F}^{\prime} \mathbf{Q}_{\mathbf{F x}}\right)
$$

implies

$$
\mathscr{C}\left(\mathbf{M} \underline{\mathbf{V}}_{12}\right) \subseteq \mathscr{C}\left(\mathbf{M} \underline{\mathbf{V F}}^{\prime} \mathbf{Q}_{\mathbf{F X}}\right)
$$

Choosing

$$
\mathbf{F}^{\prime}=\mathbf{M}(\mathbf{M V M})^{-} \mathbf{M V}_{12},
$$

yields $\mathbf{Q}_{\mathbf{F X}}=\mathbf{I}_{f}$ and

$$
\mathbf{M V F}^{\prime} \mathbf{Q}_{\mathbf{F X}}=\operatorname{MVM}(\mathbf{M V M})^{-} \mathbf{M V}_{12}=\mathbf{M V}_{12},
$$

which confirms that for any $(\mathbf{M V M})^{-}$the condition (5.5a) holds, i.e., Fy $\in \mathcal{S}\left(\boldsymbol{\varepsilon}_{*} \mid\right.$ $\mathscr{M}_{*}$ ). This conclusion could be done also by noting that

$$
\mathbf{V}_{21} \mathbf{M}(\mathbf{M V M})^{-} \mathbf{M y}=\operatorname{BLUP}\left(\varepsilon_{*} \mid \underline{M}_{*}\right) .
$$

Notice that choosing $\mathbf{F}$ as in 5.6 actually makes $\mathbf{F y}$ linearly minimal sufficient.

Now in view of (5.4), also the following must hold:

$$
\mathscr{C}\left(\mathbf{M} \underline{\mathbf{V}}_{12}\right) \subseteq \mathscr{C}\left[\mathbf{M V} \mathbf{M}(\mathbf{M V M})^{-} \mathbf{M V}_{12}\right]
$$

As (5.9) must hold for all choices of (MVM) $)^{-}$, Lemma 3.2 shows that this holds if and only if the following two conditions hold:

$$
\begin{aligned}
\mathscr{C}\left(\mathbf{M} \underline{\mathbf{V}}_{12}\right) & \subseteq \mathscr{C}\left[\mathbf{M V} \mathbf{M}(\mathbf{M V M})^{+} \mathbf{M V}_{12}\right], \\
\mathscr{C}(\mathbf{M} \underline{\mathbf{V}}) & \subseteq \mathscr{C}(\mathbf{M V}) .
\end{aligned}
$$

In light of Lemma 3.1, (5.10b) is equivalent to

$$
\mathscr{C}(\underline{\mathbf{W}}) \subseteq \mathscr{C}(\mathbf{W}) .
$$


It remains to prove the reverse relation, i.e., that 5.10a and 5.10b together imply (5.4). To do this, we write

$$
\begin{aligned}
\mathscr{C}\left(\mathbf{M} \underline{\mathbf{V}}_{12}\right) & \subseteq \mathscr{C}\left[\mathbf{M} \underline{\mathbf{V M}}(\mathbf{M V M})^{+} \mathbf{M V}_{12}\right] \\
& \subseteq \mathscr{C}\left[\mathbf{M V M}(\mathbf{M V M})^{+} \mathbf{M V F}^{\prime} \mathbf{Q}_{\mathbf{F X}}\right] \\
& =\mathscr{C}\left[\mathbf{M} \underline{\mathbf{V}} \mathbf{M}(\mathbf{M V M})^{+} \mathbf{M V M F}^{\prime} \mathbf{Q}_{\mathbf{F X}}\right] \\
& =\mathscr{C}\left(\mathbf{M} \underline{\mathbf{V}} \mathbf{M} \mathbf{P}_{\mathbf{M V M}} \mathbf{F}^{\prime} \mathbf{Q}_{\mathbf{F X}}\right) \\
& =\mathscr{C}\left(\mathbf{M} \underline{\mathbf{V}} \mathbf{M F}^{\prime} \mathbf{Q}_{\mathbf{F X}}\right) \\
& =\mathscr{C}\left(\mathbf{M} \underline{\mathbf{V}} \mathbf{F}^{\prime} \mathbf{Q}_{\mathbf{F X}}\right) .
\end{aligned}
$$

The first inclusion in (5.12) comes from (5.10a), and the second one from (5.5a); the first equality follows from $\mathbf{F}^{\prime} \mathbf{Q}_{\mathbf{F X}}=\mathbf{M F}^{\prime} \mathbf{Q}_{\mathbf{F X}}$, and the third from (b). Notice that part (d) comes from part (e) of Lemma 3.1 Thus we have completed the proof.

Using Lemma 2.2 we can write the following corollary.

Corollary 5.1. Consider the linear models $\mathscr{M}_{*}$ and $\mathscr{M}_{*}$, where $\mathscr{C}\left(\mathbf{X}_{*}^{\prime}\right) \subseteq \mathscr{C}\left(\mathbf{X}^{\prime}\right)$, and the following statements:

(a) $\mathcal{S}\left(\mathbf{X}_{*} \boldsymbol{\beta} \mid \mathscr{M}_{*}\right) \subseteq \mathcal{S}\left(\mathbf{X}_{*} \boldsymbol{\beta} \mid \mathscr{M}_{*}\right)$,

(b) $\mathcal{S}\left(\varepsilon_{*} \mid \mathscr{M}_{*}\right) \subseteq \mathcal{S}\left(\varepsilon_{*} \mid \underline{M}_{*}\right)$,

(c) $\mathcal{S}\left(\mathbf{y}_{*} \mid \mathscr{M}_{*}\right) \subseteq \mathcal{S}\left(\mathbf{y}_{*} \mid \mathscr{M}_{*}\right)$.

Then each of the two statements above imply the third one. In particular (a) and (b) imply (c), i.e., (c) holds if

(i) $\mathscr{C}\left(\mathbf{M} \underline{\mathbf{V}}_{12}\right) \subseteq \mathscr{C}\left[\mathbf{M} \underline{\mathbf{V M}}(\mathbf{M V M})^{+} \mathbf{M} \mathbf{V}_{12}\right]$,

(ii) $\mathscr{C}(\underline{\mathbf{Z}}) \subseteq \mathscr{C}\left(\underline{\mathbf{W}} \mathbf{W}^{+} \mathbf{Z}\right)$,

(iii) $\mathscr{C}(\underline{\mathbf{W}}) \subseteq \mathscr{C}(\mathbf{W})$.

It is noteworthy that it would be interesting to find a necessary and sufficient condition for (c) in Corollary 5.1. This seems to be not so easy to do and this question is postponed for a further research topic.

\section{Conclusions}

We consider the general linear model $\mathbf{y}=\mathbf{X} \boldsymbol{\beta}+\boldsymbol{\varepsilon}$, or shortly $\mathscr{M}=\{\mathbf{y}, \mathbf{X} \boldsymbol{\beta}, \mathbf{V}\}$, supplemented with the new unobservable random vector $\mathbf{y}_{*}$, coming from $\mathbf{y}_{*}=$ $\mathbf{X}_{*} \boldsymbol{\beta}+\boldsymbol{\varepsilon}_{*}$, where the expectation of $\mathbf{y}_{*}$ is $\mathbf{X}_{*} \boldsymbol{\beta}$ and the covariance matrix of $\mathbf{y}_{*}$ is known as well as the cross-covariance matrix between $\mathbf{y}_{*}$ and $\mathbf{y}$. We denote the supplemented model as $\mathscr{M}_{*}$. The misspecified supplemented model is denoted as $\underline{M}_{*}$, and the misspecification concerns only the covariance part of the setup. 
We can denote the models shortly as

$$
\begin{aligned}
& \mathscr{M}_{*}=\left\{\left(\begin{array}{c}
\mathbf{y} \\
\mathbf{y}_{*}
\end{array}\right),\left(\begin{array}{c}
\mathbf{X} \\
\mathbf{X}_{*}
\end{array}\right) \boldsymbol{\beta},\left(\begin{array}{cc}
\mathbf{V} & \mathbf{V}_{12} \\
\mathbf{V}_{21} & \mathbf{V}_{22}
\end{array}\right)\right\}, \\
& \mathscr{M}_{*}=\left\{\left(\begin{array}{c}
\mathbf{y} \\
\mathbf{y}_{*}
\end{array}\right),\left(\begin{array}{c}
\mathbf{X} \\
\mathbf{X}_{*}
\end{array}\right) \boldsymbol{\beta},\left(\begin{array}{cc}
\underline{\mathbf{V}} & \underline{\mathbf{V}}_{12} \\
\underline{\mathbf{V}}_{21} & \underline{\mathbf{V}}_{22}
\end{array}\right)\right\} .
\end{aligned}
$$

Suppose that $\mathbf{F y}$ is linearly sufficient for estimable parametric function $\mathbf{X}_{*} \boldsymbol{\beta}$ under $\mathscr{M}_{*}$. We give necessary and sufficient conditions that $\mathbf{F y}$ continues to be linearly sufficient for $\mathbf{X}_{*} \boldsymbol{\beta}$ under the model $\underline{M}_{*}$. The corresponding properties regarding the linear sufficiency with respect to $\varepsilon_{*}$ and $\mathbf{y}_{*}$ are also studied.

Consider then the mixed linear model $\mathbf{y}=\mathbf{X} \boldsymbol{\beta}+\mathbf{Z u}+\mathbf{e}$, where $\mathbf{X}_{n \times p}$ and $\mathbf{Z}_{n \times q}$ are known matrices, $\boldsymbol{\beta} \in \mathbb{R}^{p}$ is a vector of unknown fixed effects, $\mathbf{u}$ is an unobservable vector $(q$ elements) of random effects with $\mathrm{E}(\mathbf{u})=\mathbf{0}, \operatorname{cov}(\mathbf{u})=\boldsymbol{\Delta}$, e is a random error vector with $\mathrm{E}(\mathbf{e})=\mathbf{0}, \operatorname{cov}(\mathbf{e})=\boldsymbol{\Phi}$, and $\operatorname{cov}(\mathbf{e}, \mathbf{u})=\mathbf{0}$. Denoting $\mathbf{g}=\mathbf{X} \boldsymbol{\beta}+\mathbf{Z u}$, we have $\operatorname{cov}(\mathbf{y})=\operatorname{cov}(\mathbf{Z u}+\mathbf{e})=\mathbf{Z} \boldsymbol{\Delta} \mathbf{Z}^{\prime}+\boldsymbol{\Phi}=\mathbf{\Sigma}$ and

$$
\operatorname{cov}\left(\begin{array}{l}
\mathbf{y} \\
\mathbf{g}
\end{array}\right)=\operatorname{cov}\left(\begin{array}{c}
\mathbf{y} \\
\mathbf{Z u}
\end{array}\right)=\left(\begin{array}{cc}
\boldsymbol{\Sigma} & \mathbf{Z} \boldsymbol{\Delta} \mathbf{Z}^{\prime} \\
\mathbf{Z} \boldsymbol{\Delta} \mathbf{Z}^{\prime} & \mathbf{Z} \boldsymbol{\Delta} \mathbf{Z}^{\prime}
\end{array}\right)=\left(\begin{array}{cc}
\boldsymbol{\Sigma} & \boldsymbol{\Sigma}_{12} \\
\boldsymbol{\Sigma}_{21} & \boldsymbol{\Sigma}_{22}
\end{array}\right):=\boldsymbol{\Omega} .
$$

Now the mixed linear model can be expressed as a version of the model with "new observations", the new observations being in $\mathbf{g}=\mathbf{X} \boldsymbol{\beta}+\mathbf{Z u}$ :

$$
\mathscr{L}_{*}:=\left\{\left(\begin{array}{l}
\mathbf{y} \\
\mathbf{g}
\end{array}\right),\left(\begin{array}{l}
\mathbf{X} \\
\mathbf{X}
\end{array}\right) \boldsymbol{\beta},\left(\begin{array}{cc}
\boldsymbol{\Sigma} & \boldsymbol{\Sigma}_{12} \\
\boldsymbol{\Sigma}_{21} & \boldsymbol{\Sigma}_{22}
\end{array}\right)\right\}
$$

The misspecified mixed model is then $\mathscr{L}_{*}$, where the covariance matrix would be $\underline{\boldsymbol{\Omega}}$. Thus the results concerning the linear sufficiency in the misspecified mixed model can be directly obtained from the corresponding properties of the models with new observations. For the linear sufficiency in the mixed model, see also Isotalo et al. (2018) and Markiewicz \& Puntanen (2018c).

\section{Acknowledgements}

Part of this research was done during the meeting of an International Research Group on Multivariate and Mixed Linear Models in the Mathematical Research and Conference Center, Będlewo, Poland, November 2017 and March 2018, supported by the Stefan Banach International Mathematical Center.

\section{References}

Baksalary, J.K. \& Kala, R. (1981). Linear transformations preserving best linear unbiased estimators in a general Gauss-Markoff model. Ann. Stat., 9, 913-916. DOI

Baksalary, J.K. \& Kala, R. (1986). Linear sufficiency with respect to a given vector of parametric functions. J. Stat. Plan. Inf., 14, 331-338. DOI 
Baksalary, J.K. \& Mathew, T. (1986). Linear sufficiency and completeness in an incorrectly specified general Gauss-Markov model. Sankhyā Ser. A, 48, 169-180. DOI

Christensen, R. (2011). Plane Answers to Complex Questions: the Theory of Linear Models, 4th Edition. Springer, New York. DOI.

Drygas, H. (1983). Sufficiency and completeness in the general Gauss-Markov model. Sankhyā Ser. A, 45, 88-98. DOI

Haslett, S.J. \& Puntanen, S. (2017). Best linear unbiased prediction (BLUP). Wiley StatsRef: Statistics Reference Online. DOI.

Hauke, J., Markiewicz, A. \& Puntanen, S. (2012). Comparing the BLUEs under two linear models. Commun. Stat. Theory Methods, 41, 2405-2418. DOI.

Hauke, J., Markiewicz, A. \& Puntanen, S. (2013). Revisiting the BLUE in a linear model via proper eigenvectors. Combinatorial Matrix Theory and Generalized Inverses of Matrices. R.B. Bapat, S. Kirkland, K.M. Prasad \& S. Puntanen, eds. Springer, pp. 73-83. DOI.

Isotalo, J., Markiewicz, A. \& Puntanen, S. (2018). Some properties of linear prediction sufficiency in the linear model. Trends and Perspectives in Linear Statistical Inference: LinStat, Istanbul, August 2016. M. Tez \& D. von Rosen, eds. Springer, pp. 111-129. DOI.

Isotalo, J. \& Puntanen, S. (2006a). Linear sufficiency and completeness in the partitioned linear model. Acta Comment. Univ. Tartu Math., 10, 53-67.

Isotalo, J. \& Puntanen, S. (2006b). Linear prediction sufficiency for new observations in the general Gauss-Markov model. Commun. Stat. Theory Methods, 35, 1011-1023. DOI

Kala, R., Markiewicz, A. \& Puntanen, S. (2017). Some further remarks on the linear sufficiency in the linear model. Applied and Computational Matrix Analysis: MatTriad, Coimbra, Portugal, September 2015, Selected, Revised Contributions. N. Bebiano, ed. Springer Proceedings in Mathematics \& Statistics, vol. 192, pp. 275-294. DOI.

Kala, R., Puntanen, S. \& Tian, Y. (2017). Some notes on linear sufficiency. Stat. Pap., 58, $1-17$. DOI

Markiewicz, A. (1998). Comparison of linear restricted models with respect to the validity of admissible and linearly sufficient estimators. Statist. Probab. Lett., 38, 347-354. DOI

Markiewicz, A. \& Puntanen, S. (2018a). Further properties of linear prediction sufficiency and the BLUPs in the linear model with new observations. Afrika Statistika, 13, 15111530. DOI

Markiewicz, A. \& Puntanen, S. (2018b). Further properties of the linear sufficiency in the partitioned linear model. Matrices, Statistics and Big Data (Proceedings of the 25th International Workshop on Matrices and Statistics, IWMS-2016, held in Funchal, Madeira. Portugal, 6-9 June 2016). Springer, in press.

Markiewicz, A. \& Puntanen, S. (2018c). Upper bounds for the Euclidean distances between the BLUPs. Special Matrices, 6, 249-261. DOI

Mitra, S.K. \& Moore, B.J. (1973). Gauss-Markov estimation with an incorrect dispersion matrix. Sankhyā, Ser. A, 35, 139-152. DOI

Puntanen, S. \& Styan, G.P.H. (1989). The equality of the ordinary least squares estimator and the best linear unbiased estimator [with comments by O. Kempthorne \& by S.R. Searle and with "Reply" by the authors]. Amer. Statist., 43, 153-164. DOI

Puntanen, S., Styan, G.P.H. \& Isotalo, J. (2011). Matrix Tricks for Linear Statistical Models: Our Personal Top Twenty. Springer, Heidelberg. DOI

Rao, C.R. (1971). Unified theory of linear estimation. Sankhyā Ser. A, 33, 371-394. [Corrigendum (1972), 34, p. 194 and p. 477.] DOI 
Rao, C.R. (1973). Representations of best linear estimators in the Gauss-Markoff model with a singular dispersion matrix. J. Multivariate Anal., 3, 276-292. DOI

Rao, C.R. (1974). Projectors, generalized inverses and the BLUE's, J. Roy. Statist. Soc. Ser. $B, 36,442-448$. DOI

Rao, C.R. \& Mitra, S.K. (1971). Generalized Inverse of Matrices and Its Applications. Wiley, New York.

Tian, Y. (2009). On equalities for BLUEs under misspecified Gauss-Markov models. Acta Math. Sin. (English Ser.), 25, 1907-1920. DOI

Tian, Y. \& Puntanen, S. (2009). On the equivalence of estimations under a general linear model and its transformed models. Linear Algebra Appl., 430, 2622-2641. DOI

Zyskind, G. \& Martin, F.B. (1969). On best linear estimation and general Gauss-Markov theorem in linear models with arbitrary nonnegative covariance structure. SIAM J. Appl. Math., 17, 1190-1202. DOI 